\title{
水系锂离子电池负极材料 $\mathrm{LiTi}_{2}\left(\mathrm{PO}_{4}\right)_{3}$ 的研究进展
}

\author{
王禹桐 ${ }^{1}$, 张非凡 ${ }^{1}$, 许乃才 ${ }^{2}$, 王春霞 ${ }^{1}$, 崔立山 ${ }^{1}$, 黄国勇 ${ }^{1}$ \\ (1. 中国石油大学(北京) 新能源与材料学院, 重质油国家重点实验室, 北京 $102249 ; 2$. 青海师范大学 化学化工学 \\ 院, 西宁 810008)
}

摘 要: 锂离子电池作为一种绿色可充电电池, 具有较高的能量密度及功率密度, 但市售锂离子电池主要以有机物 为电解液, 当电池过充或短路时存在一定的燃烧及爆炸风险。为应对此问题, 水系锂离子电池逐渐走进人们的视野, 它具有清洁环保、安全性能高等优点, 其工作电压为 $1.5 \sim 2.0 \mathrm{~V}$, 主要应用于储能领域。考虑到水系电池的析氢析 氧反应，常规负极材料无法应用于水系锂离子电池，因此水系锂离子电池的研发关键在于负极材料的选取。 $\mathrm{LiTi}_{2}\left(\mathrm{PO}_{4}\right)_{3}$ 具有开放的三维通道以及合适的嵌锂电位, 可以作为水系锂离子电池的负极材料。 $\mathrm{LiTi}_{2}\left(\mathrm{PO}_{4}\right)_{3}$ 的合成方 法主要有高温固相法、溶胶一凝胶法和水热法等。为进一步提高 $\mathrm{LiTi}_{2}\left(\mathrm{PO}_{4}\right)_{3}$ 的电化学性能, 可以采用颗粒纳米化、 形貌控制、元素掺杂及碳包覆等方式进行改性。本文从合成方法及改性手段的角度, 对近年来国内外水系锂离子电 池负极材料 $\mathrm{LiTi}_{2}\left(\mathrm{PO}_{4}\right)_{3}$ 的研究进行综述, 并对 $\mathrm{LiTi}_{2}\left(\mathrm{PO}_{4}\right)_{3}$ 负极材料的发展前景做出展望。

关 键 词: 水系锂离子电池; 负极材料; $\mathrm{LiTi}_{2}\left(\mathrm{PO}_{4}\right)_{3}$; 综述

中图分类号: TM912 文献标志码: A

\section{Research Progress of $\mathrm{LiTi}_{2}\left(\mathrm{PO}_{4}\right)_{3}$ Anode for Aqueous Lithium-ion Batteries}

\author{
WANG Yutong ${ }^{1}$, ZHANG Feifan $^{1}$, XU Naicai ${ }^{2}$, WANG Chunxia ${ }^{1}$, CUI Lishan ${ }^{1}$, HUANG Guoyong ${ }^{1}$ \\ (1. State Key Laboratory of Heavy Oil, College of New Energy and Materials, China University of Petroleum-Beijing, Beijing \\ 102249, China; 2. School of Chemistry and Chemical Engineering, Qinghai Normal University, Xining 810008, China)
}

\begin{abstract}
As green rechargeable batteries, lithium-ion batteries feature high energy and power density. However, commonly-used electrolytes, organic compounds, in commercially available lithium-ion batteries are flammable and toxic, which leaves them at the risk of combustion and explosion when being overcharged or short-circuited. In order to solve this problem, much attention has been paid to lithium-ion batteries with aqueous electrolytes, which take low-toxicity and high safety as the prominent advantages. The working voltage, $1.5-2.0 \mathrm{~V}$, indicates their usage mainly in the field of energy storage. Considering the hydrogen and oxygen evolution, conventional anode materials used in commercially available lithium-ion batteries are inconformity for water-based lithium-ion batteries. Therefore, the key to the development of aqueous lithium-ion batteries lies in the selection of anodes. The anode material, $\mathrm{LiTi}_{2}\left(\mathrm{PO}_{4}\right)_{3}$, has drawn the attention of researchers due to its advantages such as three-dimensional channel and appropriate lithium-ion intercalation potential. The synthesis methods of $\mathrm{LiTi}_{2}\left(\mathrm{PO}_{4}\right)_{3}$ mainly include high temperature solid-phase
\end{abstract}

收稿日期: 2021-08-13; 收到修改稿日期：2021-10-22; 网络出版日期: 2021-11-01

基金项目: 国家自然科学基金(52022109, 51834008); 北京市自然科学基金(2202047); 中国石油大学(北京)科研基金 (2462018YJRC041, 2462020YXZZ016)

National Natural Science Foundation of China (52022109, 51834008); Beijing Municipal Natural Science Foundation (2202047); Science Foundation of China University of Petroleum, Beijing (2462018YJRC041, 2462020YXZZ016)

作者简介: 王禹桐(1992-)，男，博士研究生. E-mail: 1248736790@qq.com

WANG Yutong (1992-), male, PhD candidate. E-mail: 1248736790@qq.com

通信作者: 黄国勇, 教授. E-mail: huanggy@cup.edu.cn

HUANG Guoyong, professor. E-mail: huanggy@cup.edu.cn 
calcination, Sol-Gel methods and hydrothermal reaction, etc. To further improve the electrochemical performance of $\mathrm{LiTi}_{2}\left(\mathrm{PO}_{4}\right)_{3}$, strategies can be used such as particle nanocrystallization, morphology control, element doping, and carbon-coating. This review focuses on the synthesis and modification of $\operatorname{LiTi}\left(\mathrm{PO}_{4}\right)_{3}$, as well as related research progress. At last, the future development of $\mathrm{LiTi}_{2}\left(\mathrm{PO}_{4}\right)_{3}$ as anode material for lithium-ion battery is properly prospected.

Key words: aqueous lithium-ion battery; anode material; $\mathrm{LiTi}_{2}\left(\mathrm{PO}_{4}\right)_{3}$; review

锂离子电池具有能量密度大、工作电压高、循 环寿命长、无记忆效应等显著优势 ${ }^{[1-5]}$ 。然而, 锂离 子电池并非十全十美, 其安全问题亟待解决。近年 来, 电池燃烧、爆炸事故频发, 事故的主要原因之一 在于锂离子电池电解液所使用的溶剂(如碳酸乙烯 酯(Ethylene carbonate, EC); 碳酸二甲酯(Dimethyl carbonate, DMC); 碳酸二乙酯 (Diethyl carbonate, DEC)等)为易燃有机物，当电池过充或温度过高时， 便存在爆炸的风险 ${ }^{[6]}$ 。为应对当下锂离子电池的安全 问题, 科研人员开始关注水系锂离子电池。

水系锂离子电池的概念最早由加拿大的 Dahn 团队 ${ }^{[7]}$ 提出。如表 1 所示 ${ }^{[8]}$, 与有机系锂离子电池相 比, 水系锂离子电池的主要特点在于其以非可燃性 锂盐的水溶液为电解液, 可以从根源上有效避免热 失控反应，大大降低了安全隐患。此外，水溶液的离 子电导率通常较有机系电解液高出两个数量级, 有 助于提高电池的倍率性能 ${ }^{[9]}$ 。另一方面, 组装水系锂 离子电池无需严格无水条件, 其装配复杂程度低于 有机系锂离子电池, 有助于实现规模化生产。水系 锂离子电池兼顾了安全与成本优势, 具有很高的开 发潜力和应用价值 ${ }^{[7,9-10]}$ 。

构建水系电池需要考虑以下问题: 电极材料与 $\mathrm{H}_{2} \mathrm{O}$ 及 $\mathrm{O}_{2}$ 的副反应; 正极材料的质子共嵌入现象; 析氢析氧反应等 ${ }^{[11]}$ 。其中, 电极材料的副反应及质 子共嵌入现象可以通过消除 $\mathrm{O}_{2}$ 、调整电解液的 $\mathrm{pH}$ 得到改善 ${ }^{[12]}$ 。因此, 析氢析氧反应成为了构建水系 锂离子电池所需考虑的首要问题, 由于体系以水为 溶剂, 当电极材料的嵌锂电位超出水的分解电压窗 口时, 电池会发生明显的析氢析氧现象 ${ }^{[13]}$, 影响正 常使用, 因此电极材料应严格在该范围内选取。

由图 1 可知, 用于传统有机系电解液的正极材 料(如 $\mathrm{LiCoO}_{2} 、 \mathrm{LiNiO}_{2} 、 \mathrm{LiMn}_{2} \mathrm{O}_{4}$ 和 $\mathrm{LiFePO}_{4}$ 等)的
嵌锂电位低于氧气析出电位, 在水系锂离子电池中 可以充当正极活性材料。但对于负极而言, 商用负 极材料石墨的嵌锂电位过低, 无法应用于水系锂离 子电池。因此, 构建水系锂离子电池的重点就是在 稳定的电压窗口内选取合适的负极活性材料。只有 高于氢气析出电位的材料 ${ }^{[14]}$ (如 $\mathrm{LiTi}_{2}\left(\mathrm{PO}_{4}\right)_{3}$ 、 $\mathrm{TiP}_{2} \mathrm{O}_{7} 、 \mathrm{VO}_{2}$ 和 $\mathrm{LiV}_{3} \mathrm{O}_{8}$ 等)才能在水系锂离子电池体 系中充当负极。

如表 2 所示 ${ }^{[14]}$, 在众多负极材料中, $\mathrm{LiTi}_{2}\left(\mathrm{PO}_{4}\right)_{3}$ 具有嵌锂电位适宜、比容量较大及骨架稳定等优势。 本文将对 $\mathrm{LiTi}_{2}\left(\mathrm{PO}_{4}\right)_{3}$ 的制备方法及改性手段等进行 综述。

\section{1 磷酸钛锂的结构及嵌锂行为}

$\mathrm{LiTi}_{2}\left(\mathrm{PO}_{4}\right)_{3}$ 属于菱方晶系, 空间群为 $\mathrm{R}-3 \mathrm{c}$, 离子电 导率为 $2.0 \times 10^{-6} \mathrm{~S} \cdot \mathrm{cm}^{-1}$, 理论比容量为 $138 \mathrm{mAh} \cdot \mathrm{g}^{-1}$ 。 晶格中 3 个 $\mathrm{PO}_{4}$ 四面体和 2 个 $\mathrm{TiO}_{6}$ 八面体通过共用 顶点 $\mathrm{O}$ 原子连接而形成 $\left[\mathrm{Ti}_{2}\left(\mathrm{PO}_{4}\right)_{3}\right]^{-1}$ 菱面体 ${ }^{[15-16]}$, 如 图 2 所示 ${ }^{[17]}$ 。晶格中 $\mathrm{P}-\mathrm{O}$ 共价键具有较大的键能, 使

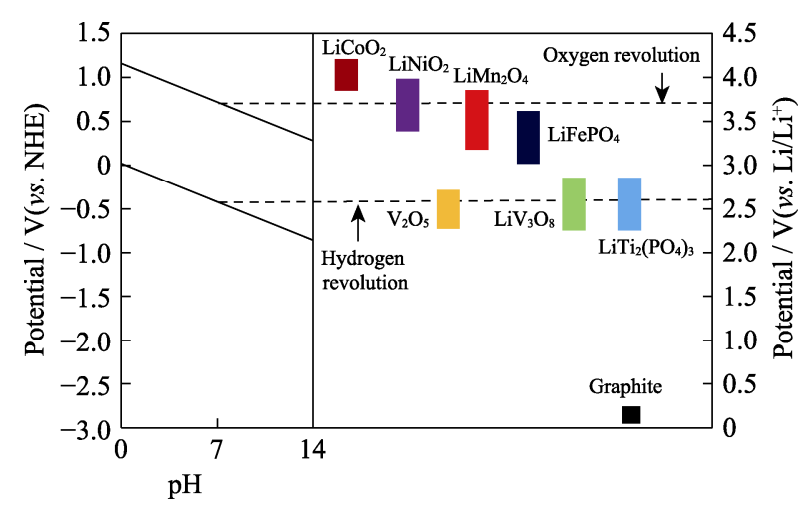

图 1 电极材料的嵌锂电位-pH 的关系图

Fig. 1 Potential-pH diagram of typical electrode materials

表 1 水系锂离子电池和有机系锂离子电池的特征比较 ${ }^{[8]}$

Table 1 Comparison of the characteristics of aqeuous and organic lithium-ion batteries ${ }^{[8]}$

\begin{tabular}{cccccc}
\hline Type & Operating voltage/V & Safety & Electrolyte & Solvent & Cost \\
\hline Organic Li-ion battery & $3.6-4.2$ & Low & $\mathrm{LiPF}_{6}, \mathrm{LiAsF}_{6}$, etc & $\mathrm{EC}, \mathrm{DMC}, \mathrm{DEC}$, etc & $\mathrm{High}$ \\
Aqeuous Li-ion battery & $1.5-2.0$ & $\mathrm{High}$ & $\mathrm{Li}_{2} \mathrm{SO}_{4}, \mathrm{LiNO}_{3}$, etc & $\mathrm{H}_{2} \mathrm{O}$ & Moderate \\
\hline
\end{tabular}


表 2 部分水系锂离子电池的负极材料的参数 ${ }^{[14]}$

Table 2 Parameters of some anode materials for aqeuous lithium-ion battery ${ }^{[14]}$

\begin{tabular}{lc}
\hline Anode material & $\begin{array}{c}\text { Specific capacity/ } \\
\left(\mathrm{mAh} \cdot \mathrm{g}^{-1}\right)\end{array}$ \\
\hline $\mathrm{LiTi}_{2}\left(\mathrm{PO}_{4}\right)_{3}$ & 138 \\
$\mathrm{TiP}_{2} \mathrm{O}_{7}$ & 121 \\
$\mathrm{VO}_{2}$ & 250 \\
$\mathrm{LiV}_{3} \mathrm{O}_{8}$ & 250 \\
\hline &
\end{tabular}

图 $2 \mathrm{LiTi}_{2}\left(\mathrm{PO}_{4}\right)_{3}$ 的晶体结构图 ${ }^{[17]}$

Fig. 2 Crystal structure of $\mathrm{LiTi}_{2}\left(\mathrm{PO}_{4}\right)_{3}{ }^{[17]}$

得 $\mathrm{O}$ 原子在充放电过程中难以析出, 从而确保了晶 体结构的稳定性。此外, 引入 $\mathrm{P}$ 导致 Ti-O 键的离子 键特性突出, 增大了反键轨道与锂的费米能级差, 从而使 $\mathrm{Ti}^{4+} / \mathrm{Ti}^{3+}$ 电对具有较高的氧化还原电位 $(\sim 2.5 \mathrm{~V}$ $\left.\left(v s \mathrm{Li} / \mathrm{Li}^{+}\right)\right)^{[18]}$, 符合水系锂离子电池对负极材料的 电位要求。

$\mathrm{LiTi}_{2}\left(\mathrm{PO}_{4}\right)_{3}$ 三维刚性骨架中存在两种 $\mathrm{Li}$ 间隙位 点, 分别记作 M1 和 M2。M1 位于沿 $z$ 轴方向上两 个相邻的 $\mathrm{TiO}_{6}$ 八面体间, $\mathrm{M} 2$ 位点则处在

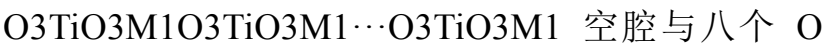
原子键合形成的导电通道拐弯处。由于 M1 位点具 有较低的势能, 因此在 $\mathrm{LiTi}_{2}\left(\mathrm{PO}_{4}\right)_{3}$ 晶格中, $\mathrm{Li}^{+}$占据 M1 位点, 而 M2 位点则处于空腔状态。在嵌锂过程 中, 来自正极的 $\mathrm{Li}^{+}$开始占据晶格中的 $\mathrm{M} 2$ 位点, 与 此同时, 处于 $\mathrm{M} 1$ 位点的 $\mathrm{Li}^{+}$也开始逐渐迁移至 M2 位点 ${ }^{[15,19]}$, 为满足电荷守恒, $\mathrm{Ti}$ 的价态由 +4 降低至

Potential/

$\mathrm{V}(v s$. NHE)

$-0.5 \quad$ Moderate specific capacity, stable framework

-0.4 Low specific capacity, high Li-intercalation potential

$-0.4 \quad$ High specific capacity, poor cycling performance

$-0.4 \quad$ Being fragile during cycling
+3 , 形成 $\mathrm{Li}_{3} \mathrm{Ti}_{2}\left(\mathrm{PO}_{4}\right)_{3}$ 相。

$$
\mathrm{LiTi}_{2}\left(\mathrm{PO}_{4}\right)_{3}+2 \mathrm{e}^{-}+2 \mathrm{Li}^{+} \rightarrow \mathrm{Li}_{3} \mathrm{Ti}_{2}\left(\mathrm{PO}_{4}\right)_{3}
$$

在 $\mathrm{Li}_{3} \mathrm{Ti}_{2}\left(\mathrm{PO}_{4}\right)_{3}$ 晶格中, 3 个 $\mathrm{Li}^{+}$均匀分散在 $\mathrm{M} 2$ 位点中, 而 $M 2$ 位点可进一步划分为 M3 和 M3'亚位 点, 两个 $\mathrm{Li}^{+}$占据 $\mathrm{M} 3$ 位点 $(2 / 3)$, 另一个 $\mathrm{Li}^{+}$占据 $\mathrm{M} 3{ }^{\prime}$ 位点 $(1 / 3)^{[15]}$ 。由此可见，充放电过程仅涉及 $\mathrm{Li}^{+}$位点 的重排，而晶格体积不发生明显变化，保证了其循 环使用的稳定性和安全性。

\section{2 合成方法}

目前, 文献报道的 $\mathrm{LiTi}_{2}\left(\mathrm{PO}_{4}\right)_{3}$ 合成方法主要有 高温固相法、溶胶-凝胶法、水/溶剂热法及液相共 沉淀法等，这些方法的特点如表 3 所示。

\section{1 高温固相法}

高温固相法, 即将锂源、钛源和磷源按化学计 量比混合均匀后直接置于加热炉中㷽烧, 之后经冷 却、研磨, 即可获得 $\mathrm{LiTi}_{2}\left(\mathrm{PO}_{4}\right)_{3}$ 粉末。高温固相法 的最大优势在于工艺流程简单、易于工业化生产, 缺点在于煅烧温度高、时间长, 而长时间的高温煅 烧以及较差的原料混合程度导致产物的粒径尺寸较 大且均一度差, 较大的粒径尺寸使 $\mathrm{Li}^{+}$的扩散路径 延长, 且增加了阻力, 最终影响产物的电化学性能。 此外, 高温固相法合成 $\mathrm{LiTi}_{2}\left(\mathrm{PO}_{4}\right)_{3}$ 的温度通常在 $900{ }^{\circ} \mathrm{C}$ 以上 ${ }^{[20,25]}$, 此温度下锂元素易挥发, 需要在 物料中添加过量的锂盐, 从而使得配方难以控制, 产品中容易出现杂相。

表 3 常见 $\operatorname{LiTi}_{2}\left(\mathbf{P O}_{4}\right)_{3}$ 合成方法比较

Table 3 Comparison of common synthetic methods of $\mathrm{LiTi}_{2}\left(\mathrm{PO}_{4}\right)_{3}$

\begin{tabular}{|c|c|c|c|c|c|c|}
\hline \multirow{2}{*}{ Method } & \multicolumn{3}{|c|}{ Starting materials } & \multirow{2}{*}{$\begin{array}{l}\text { Product } \\
\text { morphology }\end{array}$} & \multirow{2}{*}{ Features } & \multirow{2}{*}{ Ref. } \\
\hline & Li source & Ti source & P source & & & \\
\hline Solid state & $\mathrm{LiH}_{2} \mathrm{PO}_{4}$ & $\mathrm{TiO}_{2}$ & $\mathrm{NH}_{4} \mathrm{H}_{2} \mathrm{PO}_{4}$ & Irregular particles & Long calcination time, high temperature & {$[20]$} \\
\hline Sol-Gel & $\mathrm{CH}_{3} \mathrm{COOLi}$ & $\mathrm{Ti}\left(\mathrm{C}_{4} \mathrm{H}_{9} \mathrm{O}\right)_{4}$ & $\mathrm{H}_{3} \mathrm{PO}_{4}$ & Particles & Short calcination time, low temperature & {$[21]$} \\
\hline Hydrothermal synthesis & $\mathrm{CH}_{3} \mathrm{COOLi}$ & $\mathrm{Ti}\left(\mathrm{C}_{4} \mathrm{H}_{9} \mathrm{O}\right)_{4}$ & $\mathrm{NH}_{4} \mathrm{H}_{2} \mathrm{PO}_{4}$ & Regular particles & $\begin{array}{l}\text { Regular particle morphology, great crys- } \\
\text { tallinity }\end{array}$ & {$[22]$} \\
\hline Co-precipitation method & $\mathrm{LiOH}$ & $\operatorname{Ti}\left(\mathrm{C}_{4} \mathrm{H}_{9} \mathrm{O}\right)_{4}$ & $\mathrm{H}_{3} \mathrm{PO}_{4}$ & Particles & Requiring precise control & {$[23]$} \\
\hline Electrospinning & $\mathrm{CH}_{3} \mathrm{COOLi}$ & $\mathrm{Ti}\left(\mathrm{C}_{4} \mathrm{H}_{9} \mathrm{O}\right)_{4}$ & $\mathrm{NH}_{4} \mathrm{H}_{2} \mathrm{PO}_{4}$ & Fiber & $\begin{array}{l}\text { Ideal electrochemical performance, being } \\
\text { industrialization }\end{array}$ & [24] \\
\hline
\end{tabular}


中国科学院物理研究所陈立泉院士团队 ${ }^{[20]}$ 研究 了 $\mathrm{LiTi}_{2}\left(\mathrm{PO}_{4}\right)_{3}$ 作为水系锂电池负极材料的性能。他们 将化学计量比的 $\mathrm{LiH}_{2} \mathrm{PO}_{4} 、 \mathrm{TiO}_{2}$ 和 $\mathrm{NH}_{4} \mathrm{H}_{2} \mathrm{PO}_{4}$ 充分 混合并球磨 $5 \mathrm{~h}$, 然后升温至 $300{ }^{\circ} \mathrm{C}$ 并恒温 $4 \mathrm{~h}$, 冷 却至室温后再球磨 $2 \mathrm{~h}$, 随后升温至 $600{ }^{\circ} \mathrm{C}$, 恒温 $24 \mathrm{~h}$, 最后将样品升温至 $1000{ }^{\circ} \mathrm{C}$ 恒温 $48 \mathrm{~h}$ 得到纯 相 $\mathrm{LiTi}_{2}\left(\mathrm{PO}_{4}\right)_{3}$ 。该团队将纯相 $\mathrm{LiTi}_{2}\left(\mathrm{PO}_{4}\right)_{3}$ 作为负极 材料, 并以 $\mathrm{LiMn}_{2} \mathrm{O}_{4}$ 为正极材料, $5 \mathrm{~mol} \cdot \mathrm{L}^{-1} \mathrm{LiNO}_{3}$ 溶液为电解液, 组装全电池, 其初始放电比容量约 为 $45 \mathrm{mAh} \cdot \mathrm{g}^{-1}$ 。该全电池的比容量衰减较快, 在 $0.1 C$ 倍率下, 经 10 次充放电循环后, 放电比容量保 持率降至 $75 \%$, 这可能是源于充放电过程中晶体结 构分解和新物质生成 ${ }^{[26-27]}$ 。郑威 ${ }^{[28]}$ 采用相同的原料 制备了纯相 $\mathrm{LiTi}_{2}\left(\mathrm{PO}_{4}\right)_{3}$, 但煅烧方案非常简单: 在 $950{ }^{\circ} \mathrm{C}$ 下恒温 $12 \mathrm{~h}$, 并采用非原位 X 射线衍射(X-ray diffraction, XRD) 和热重-差热分析 (Thermogravimetric-differential thermal analysis, TG-DTA)探究了 $\mathrm{LiTi}_{2}\left(\mathrm{PO}_{4}\right)_{3}$ 合成机理，涉及的化学反应如下:

$$
\begin{aligned}
2 \mathrm{H}_{3} \mathrm{PO}_{4}+\mathrm{TiO}_{2} & \rightarrow \mathrm{TiP}_{2} \mathrm{O}_{7}+3 \mathrm{H}_{2} \mathrm{O} \\
\mathrm{LiH}_{2} \mathrm{PO}_{4} & \rightarrow \mathrm{LiPO}_{3}+\mathrm{H}_{2} \mathrm{O}
\end{aligned}
$$$$
\mathrm{TiP}_{2} \mathrm{O}_{7}+\mathrm{TiO}_{2}+\mathrm{LiPO}_{3} \rightarrow \mathrm{LiTi}_{2}\left(\mathrm{PO}_{4}\right)_{3}
$$

Wang 等 ${ }^{[25]}$ 以 $\mathrm{Li}_{2} \mathrm{CO}_{3} 、 \mathrm{TiO}_{2}$ 和 $\mathrm{NH}_{4} \mathrm{H}_{2} \mathrm{PO}_{4}$ 为原 料合成了 $\mathrm{LiTi}_{2}\left(\mathrm{PO}_{4}\right)_{3}$ 。首先在 $900{ }^{\circ} \mathrm{C}$ 下保温 $12 \mathrm{~h}$ 以分解 $\mathrm{Li}_{2} \mathrm{CO}_{3}$ 和 $\mathrm{NH}_{4} \mathrm{H}_{2} \mathrm{PO}_{4}$, 冷却至室温后取出样 品并研磨, 最后在 $1250{ }^{\circ} \mathrm{C}$ 下轱烧 $24 \mathrm{~h}$, 煅烧过程中 总化学反应如下:

$$
\begin{array}{r}
0.5 \mathrm{Li}_{2} \mathrm{CO}_{3}+2 \mathrm{TiO}_{2}+3 \mathrm{NH}_{4} \mathrm{H}_{2} \mathrm{PO}_{4} \rightarrow \\
\mathrm{LiTi}_{2}\left(\mathrm{PO}_{4}\right)_{3}+3 \mathrm{NH}_{3}+4.5 \mathrm{H}_{2} \mathrm{O}+0.5 \mathrm{CO}_{2}
\end{array}
$$

冯传启等 ${ }^{[29]}$ 对固相法稍加改进, 在原料 $\mathrm{Li}_{2} \mathrm{CO}_{3}$ 、 $\mathrm{TiO}_{2}$ 和 $\mathrm{NH}_{4} \mathrm{H}_{2} \mathrm{PO}_{4}$ 中加入适量草酸和柠檬酸溶液, 将原料调制成流变态混合物的形式进行煅烧, 于 $600{ }^{\circ} \mathrm{C}$ 下恒温 $48 \mathrm{~h}$ 获得目标产物。在流变相前驱体 中各反应组分的混合更加均匀, 能量交换更容易进 行 $^{[30]}$ 。在 $300 \mathrm{~mA} \cdot \mathrm{g}^{-1}$ 的电流密度下, 经 120 次循环 后, 比容量为 $80 \mathrm{mAh} \cdot \mathrm{g}^{-1}$ 。

\section{2 溶胶-凝胶法}

溶胶一凝胶法是目前实验室中使用频率最高的 合成 $\mathrm{LiTi}_{2}\left(\mathrm{PO}_{4}\right)_{3}$ 的方法, 它可以实现分子级别的组 分混匀, 因此与高温固相法相比, 该方法所需的煅 烧温度更低, 时间更短, 产物粒径也更均一, 电化 学性能通常优于固相法。但是, 溶胶-凝胶法的工序 较固相法复杂, 不易于扩大生产。

复旦大学夏永姚课题组 ${ }^{[31]}$ 通过溶胶-凝胶法合 成了负极材料 $\mathrm{LiTi}_{2}\left(\mathrm{PO}_{4}\right)_{3}$ 。他们将化学计量比为 1:2:3 的 $\mathrm{Li}_{2} \mathrm{CO}_{3} 、 \mathrm{TiO}_{2}$ 和 $\mathrm{NH}_{4} \mathrm{H}_{2} \mathrm{PO}_{4}$ 投入到 $2 \%$ 聚乙
烯醇(PVA)溶液中, 在 $80{ }^{\circ} \mathrm{C}$ 下, 恒温摚拌混合物直 至水分完全蒸发得到白色前驱体, 之后将其置于 $\mathrm{N}_{2}$ 气氛下 $900{ }^{\circ} \mathrm{C}$ 敾烧 $12 \mathrm{~h}$ 获得目标产物, 惰性气氛有 助于提供氧空位, 从而提高 $\mathrm{Li}^{+}$的扩散速率, 降低电 荷转移阻抗 ${ }^{[32-33]}$ 。该课题组对比了相同原料及绠烧 条件下，溶胶一凝胶法和高温固相法所得产物颗粒 的微观差异, 实验结果表明, 溶胶一凝胶法产物的平 均粒径为 200 300 nm, 而高温固相法的颗粒尺寸较 大且粒径分布不均匀。程承 ${ }^{[34]}$ 将相同的原料加入至 $2 \%$ 聚乙二醇 $(\mathrm{PEG})$ 溶液中, 搅拌并蒸发溶剂后获得 了乳白色凝胶, 最后在 $800{ }^{\circ} \mathrm{C}$ 㷽烧 $20 \mathrm{~h}$ 获得目标产 物, 粒径约为 $1 \mu \mathrm{m}$ 。在 $0.1 C$ 倍率下, 其全电池体系 $\mathrm{LiTi}_{2}\left(\mathrm{PO}_{4}\right)_{3}\left|\mathrm{Li}_{2} \mathrm{SO}_{4}\right| \mathrm{LiMn}_{2} \mathrm{O}_{4}$ 经 20 次循环后放电容量 的保持率为 $39.9 \%$ 。一些学者在溶胶体系中引入柠 檬酸和乙二醇, 利用其螯合及聚酯化反应定量生成 树脂，以使 $\mathrm{Ti}$ 原子固定在其中，从而确保各组分的 均匀分散程度，使得产物粒径分布更加均匀。 Mariappan 等 ${ }^{[35]}$ 以 $\mathrm{Li}_{2} \mathrm{CO}_{3} 、 \mathrm{NH}_{4} \mathrm{H}_{2} \mathrm{PO}_{4}$ 和钛粉为原 料, 通过此法合成了前驱体, 经 $850{ }^{\circ} \mathrm{C}$ 煅烧 $2 \mathrm{~h}$ 得 到目标产物, 粒径在 50 125 nm, 但钛粉价格昂贵 且分散性差。Wessells 和崔屹等 ${ }^{[36]}$ 以 $\mathrm{Ti}\left(\mathrm{C}_{3} \mathrm{H}_{7} \mathrm{O}\right)_{4}$ 代 替 $\mathrm{Ti}$ 粉, 在 $800{ }^{\circ} \mathrm{C}$ 煅烧 $12 \mathrm{~h}$ 获得了 $\mathrm{LiTi}_{2}\left(\mathrm{PO}_{4}\right)_{3}$, 探 究目标产物在 $\mathrm{Li}_{2} \mathrm{SO}_{4}$ 溶液中的电性能。在 $0.2 \mathrm{C}$ 倍 率下, 初始放电比容量为 $113 \mathrm{mAh} \cdot \mathrm{g}^{-1}$, 经 100 次循 环, 放电比容量约为 $101 \mathrm{mAh} \cdot \mathrm{g}^{-1}$ 。他们认为容量 保持率降低的原因可能在于 $\mathrm{LiTi}_{2}\left(\mathrm{PO}_{4}\right)_{3}$ 颗粒之间的 电接触减弱, 而充放电循环后, 材料的结晶度未见 变化。

Zhou 等 ${ }^{[37-38]}$ 以聚碳酸酯膜为模板, 将其置于 $\mathrm{LiTi}_{2}\left(\mathrm{PO}_{4}\right)_{3}$ 的前驱体溶胶中浸泡 $3 \mathrm{~h}$, 随后将聚碳酸 酯模板取出, 紧贴在集流体铂片上, 在空气气氛 $600{ }^{\circ} \mathrm{C}$ 恒温 $5 \mathrm{~h}$, 获得了生长于集流体上的单晶 $\mathrm{LiTi}_{2}\left(\mathrm{PO}_{4}\right)_{3}$ 纳米线材料。所得材料可直接作为电池的 组件使用, 避免了引入黏结剂和导电剂, 其工艺路 线如图 3 所示。纳米尺度的线状结构优势明显, 在充 放电过程中可以承受更大的应力, 防止电极材料发 生破碎。但由于体系中不存在导电剂, 其电化学性能 较差。

\section{3 水/溶剂热法}

水/溶剂热法利用体系在高温下的自生压力实 现晶粒生长，其优势在于所制备的晶粒发育相对完 整、粒径尺寸较小且分布均匀 ${ }^{[39-42]}$ 。Yue 等 ${ }^{[43]}$ 利用 水热法合成了 $\mathrm{LiTi}_{2}\left(\mathrm{PO}_{4}\right)_{3}$, 他们将 $\mathrm{Li}_{2} \mathrm{O} 、 \mathrm{TiO}_{2}$ 和 $\mathrm{P}_{2} \mathrm{O}_{5}$ 置于反应釜中, $250{ }^{\circ} \mathrm{C}$ 保温 $5 \sim 7 \mathrm{~d}$ 制备了立方形 态的目标产物, 其粒径在 $40 \sim 60 \mu \mathrm{m}$ 。 Liang 等 ${ }^{[44]}$ 


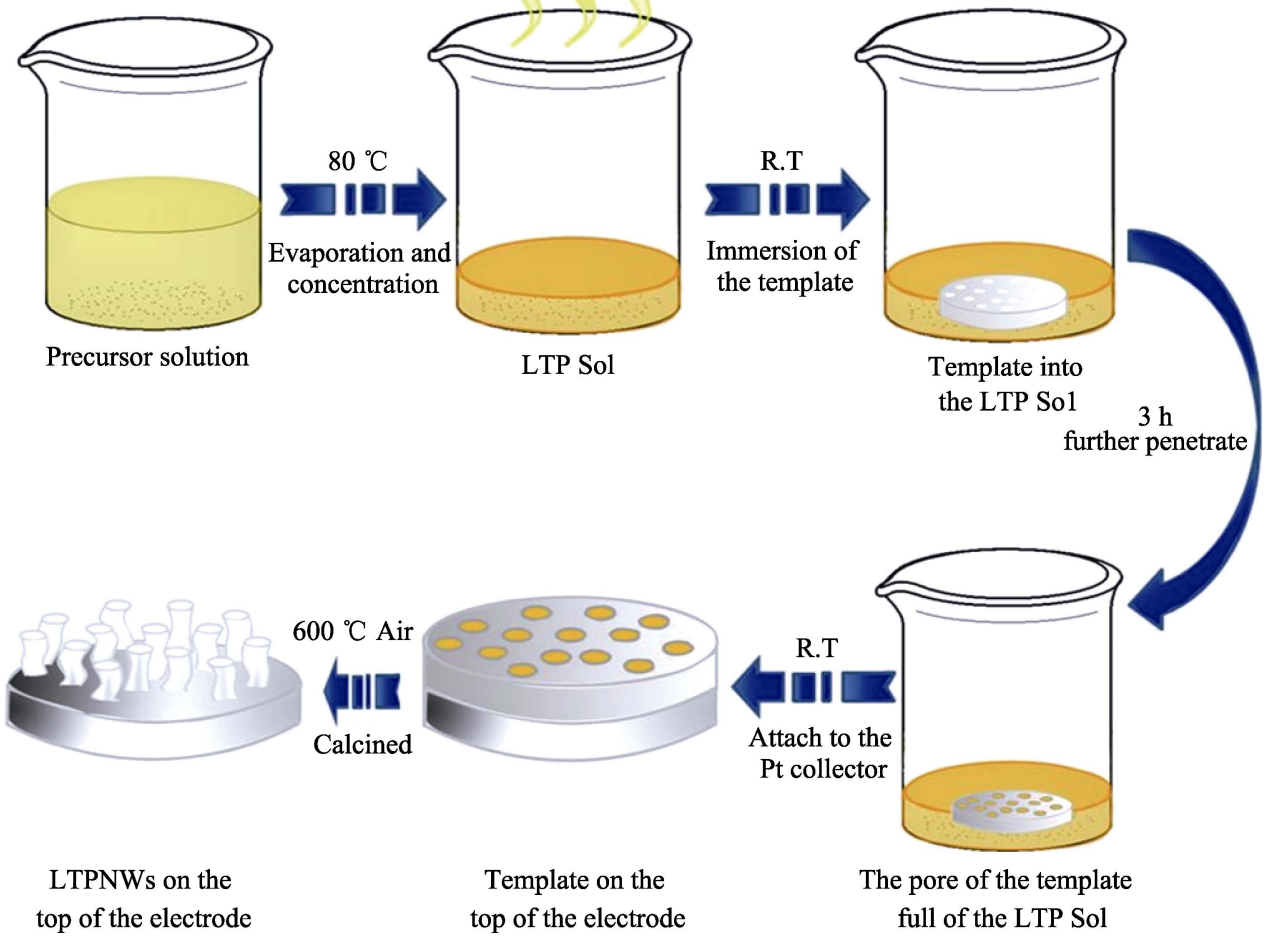

图 $3 \mathrm{LiTi}_{2}\left(\mathrm{PO}_{4}\right)_{3}$ 纳米线(LTPNMs)的制备过程示意图 ${ }^{[38]}$

Fig. 3 Schematical illustration of the fabrication process of lithium titanium phosphate nanowires (LTPNMs) ${ }^{[38]}$

以 $\mathrm{LiOH} 、 \mathrm{H}_{3} \mathrm{PO}_{4}$ 和钣粉为原料, 钽片为基板, 在 $220{ }^{\circ} \mathrm{C}$ 恒温 $24 \mathrm{~h}$ 获得了薄膜电极材料。但 Yue 等和 Liang 等均未对产物进行深入的电化学性能研究。 $\mathrm{Li}$ 等 ${ }^{[45]}$ 采用水热法, 以 $\mathrm{CH}_{3} \mathrm{COOLi} 、 \mathrm{Ti}\left(\mathrm{C}_{4} \mathrm{H}_{9} \mathrm{O}\right)_{4}$ 和 $\mathrm{NH}_{4} \mathrm{H}_{2} \mathrm{PO}_{4}$ 为原料, 经 $180{ }^{\circ} \mathrm{C}$ 恒温 $12 \mathrm{~h}$ 获得 $\mathrm{LiTi}_{2}\left(\mathrm{PO}_{4}\right)_{3}$ 。目标 产物在 $\mathrm{LiPF}_{6}(\mathrm{EC}: \mathrm{DMC}=1: 1)$ 电解液中展现出优 异的电化学性能, 在 $10 C$ 倍率下, 其初始放电比容 量为 $98.6 \mathrm{mAh} \cdot \mathrm{g}^{-1}$, 经 2000 次循环后其容量保持率 仍为 $85 \%$ 。Yu 等 ${ }^{[22]}$ 对比了原料相同时, 溶剂热法和 溶胶一凝胶法所得产物的形貌及电性能差异。溶剂热 法所得产物呈纺锤状, 在 $1 C$ 倍率下，首次放电比容 量为 $133.8 \mathrm{mAh} \cdot \mathrm{g}^{-1}$, 循环 50 次后放电比容量为 $107.6 \mathrm{mAh} \cdot \mathrm{g}^{-1}$ 。而溶胶-凝胶法所得产物则无规则形 状, $1 C$ 倍率下的初始放电比容量为 $123.7 \mathrm{mAh} \cdot \mathrm{g}^{-1}$, 50 次充放电循环后放电比容量为 $90.2 \mathrm{mAh} \cdot \mathrm{g}^{-1}$, 其 电性能略低于溶剂热法所得产物。

\section{4 共沉淀法}

共沉淀法 ${ }^{[46-48]}$ 是利用几种阳离子相近的溶解度, 使之同时形成沉淀的一种方法。该方法兼具产物颗 粒小和易工业化生产的优势, 缺点在于滴加原料时 必须保持缓慢匀速, 否则将导致产物粒径不均一。 盖利刚等 ${ }^{[49}$ 将 $\mathrm{Ti}\left(\mathrm{C}_{4} \mathrm{H}_{9} \mathrm{O}\right)_{4}$ 加入丁醇和乙醇(体积比 $1: 1)$ 的混合溶液中, 逐滴加入 $\mathrm{LiOH}$ 和植酸并持续
搅拌获得悬浊液, 之后在室温下继续摚拌 $2 \mathrm{~h}$, 对悬 浊液进行抽滤、洗涤、干燥和煓烧, 获得目标产物 $\mathrm{LiTi}_{2}\left(\mathrm{PO}_{4}\right)_{3}$ 。其全电池体系 $\mathrm{LiTi}_{2}\left(\mathrm{PO}_{4}\right)_{3} \mid 1 \mathrm{~mol} \cdot \mathrm{L}^{-1}$ $\mathrm{Li}_{2} \mathrm{SO}_{4} \mid \mathrm{LiMn}_{2} \mathrm{O}_{4}$ 在 $1.37 C$ 倍率下, 经 1000 周循环, 容量保持率仍为 $71.5 \%$ 。但 $\mathrm{Ti}\left(\mathrm{C}_{4} \mathrm{H}_{9} \mathrm{O}\right)_{4}$ 价格较高, 且 遇空气极易水解变质, 咜存成本高, 工业化的难度 增加。

\section{5 微波加热法}

微波加热法的主要优势在于加热均匀、加热速 度快、无滞后效应和温度梯度可控，因而结晶度好， 粒径均一, 理论上微波加热法可以有效抑制颗粒团 聚现象 ${ }^{[50-53]}$ 。郭晓伟等 ${ }^{[54]}$ 采用溶胶-凝胶法合成 $\mathrm{LiTi}_{2}\left(\mathrm{PO}_{4}\right)_{3}$ 前驱体, 之后将其置于微波加热炉中, 在 $600{ }^{\circ} \mathrm{C}$ 下, 仅保温 $15 \mathrm{~min}$ 便制得纯相 $\mathrm{LiTi}_{2}\left(\mathrm{PO}_{4}\right)_{3}$, 用 时远短于其他所有方法。但微波加热法的相关文献报 道较少, 因此需要积累其最佳工艺参数。此外, 微波加 热设备价格昂贵，一定程度上限制了其大规模应用。

\section{3 改性手段}

如前文所述, $\mathrm{LiTi}_{2}\left(\mathrm{PO}_{4}\right)_{3}$ 本身具有骨架稳定、嵌 锂电位适宜等优势，但是在 $\mathrm{LiTi}_{2}\left(\mathrm{PO}_{4}\right)_{3}$ 晶格中 $\mathrm{TiO}_{6}$ 八面体被 $\mathrm{PO}_{4}$ 基团分隔开, 导致波函数重叠较小, 
使得材料的电子电导率偏低。另一方面, 水系锂离 子电池通常以 $\mathrm{Li}_{2} \mathrm{SO}_{4}$ 或 $\mathrm{LiNO}_{3}$ 为电解质, 无法参与 形成 SEI 膜, 导致纯相电极材料裸露于电解液中, 加剧了电池比容量的衰减。为解决以上问题, 研究 者采用了多种方法对 $\mathrm{LiTi}_{2}\left(\mathrm{PO}_{4}\right)_{3}$ 进行改性处理, 取 得了一定进展。目前常见的改性手段可以归纳为颗 粒纳米化、微观形貌控制、碳包覆和元素掺杂等。

\section{1 颗粒纳米化及形貌控制}

颗粒纳米化是提升电极材料电化学性能的重要 思路 ${ }^{[55-59]}$ 。当材料粒径达到纳米尺度时, 能够有效缩 短 $\mathrm{Li}^{+}$的扩散路径，同时抑制缺陷的阻碍作用，控制 极化现象, 另一方面, 纳米尺度的材料具有较大的 比表面积, 可以增大电极/电解液界面, 进而增加反 应活性位点, 因此颗粒纳米化可以提高材料的倍率 性能和可逆容量 ${ }^{[60]}$ 。纳米尺度的 $\mathrm{LiTi}_{2}\left(\mathrm{PO}_{4}\right)_{3}$ 颗粒主 要通过溶胶一凝胶法、水热法等液相方法合成。

颗粒形貌控制是提高电极材料电性能的另一重 要思路。为获得具有特定形貌的颗粒往往需要采用 水/溶剂热法、静电纺丝法和模板法。Liang 等 ${ }^{[44]}$ 采用 水热法, 在 $220{ }^{\circ} \mathrm{C}$ 下保温 $48 \mathrm{~h}$ 合成了具有规则立方 形貌的 $\mathrm{LiTi}_{2}\left(\mathrm{PO}_{4}\right)_{3}$, 随着时间延长和温度升高, 颗粒 的微观形貌逐渐呈现规则的方块形状, 最大颗粒接 近 $50 \mu \mathrm{m}$ 。Deng 等 ${ }^{[61]}$ 采用溶剂热法合成了具有规则 立方形貌的 $\mathrm{LiTi}_{2}\left(\mathrm{PO}_{4}\right)_{3}$, 其粒径尺寸约为 $200 \mathrm{~nm}$ 。 在 $10 \mathrm{C}$ 倍率下, 其全电池体系 $\mathrm{LiTi}_{2}\left(\mathrm{PO}_{4}\right)_{3} \mid 1 \mathrm{~mol} \cdot \mathrm{L}^{-1}$ LiTFSI $\mid \mathrm{LiMn}_{2} \mathrm{O}_{4}$ 经 1000 次循环, 容量保持率约为 $80 \%$ 。 Liu 等 ${ }^{[24]}$ 将原料置于静电纺丝仪中, 获得了纤 维状前驱体, 经煅烧后获得 $\mathrm{LiTi}_{2}\left(\mathrm{PO}_{4}\right)_{3}$ 纤维材料, 其流程如图 4(a)所示。

与相应的颗粒材料相比, 纤维材料具有比表面 积大和长径比高等优点, 能够有效缩短 $\mathrm{Li}^{+}$的迁移 距离, 增大材料和电解液的接触面积, 有利于电化 学反应, 展现出更优秀的倍率性能, 由图 4(b)可知,
纤维材料的倍率性能明显优于相应的颗粒材料。

\section{2 碳包覆}

碳包覆是提升电极材料电性能的最常用手段之 - ${ }^{[62-65]}$, 该方法的优势在于操作简便、原料来源丰富 和提升效果显著。

\subsection{1 碳包覆提升 $\operatorname{LiTi}_{2}\left(\mathrm{PO}_{4}\right)_{3}$ 电化学性能的机制}

包覆碳层与电极材料之间为面与面的接触, 电 子更容易在活性材料和集流体中穿梭。此外, 碳包覆 可以有效抑制颗粒长大 ${ }^{[66]}$, 从而获得粒径较小的产 物。另一方面, 碳层还可以增大材料的放电比容量, 在 $\mathrm{LiTi}_{2}\left(\mathrm{PO}_{4}\right)_{3} / \mathrm{C}$ 界面, 存在 $\mathrm{C}-\mathrm{O}$ 基团, 最外端悬空 的 $\mathrm{O}$ 可以结合一部分 $\mathrm{Li}$, 从而增大比容量。相比之下, 未包覆碳层时, $\mathrm{LiTi}_{2}\left(\mathrm{PO}_{4}\right)_{3}$ 表面不存在 $\mathrm{C}-\mathrm{O}-\mathrm{Ti} / \mathrm{Li}$ 键, 表面的悬空原子如 $\mathrm{Ti} 、 \mathrm{Li}$ 和 $\mathrm{O}$ 会发生自钝化, 因此 悬空 $\mathrm{O}$ 不会与额外的 $\mathrm{Li}$ 成键 ${ }^{[61]}$ 。除此之外, 表面的 纳米级碳层阻隔了 $\mathrm{LiTi}_{2}\left(\mathrm{PO}_{4}\right)_{3}$ 与电解液的直接接触, 能够有效阻止放电态的 $\mathrm{Li}_{3-x} \mathrm{Ti}_{2}\left(\mathrm{PO}_{4}\right)_{3}$ 被 $\mathrm{O}_{2}$ 及 $\mathrm{H}_{2} \mathrm{O}$ 氧化成 $\mathrm{LiTi}_{2}\left(\mathrm{PO}_{4}\right)_{3}$ 和其他不纯相, 从而延缓电池容 量的衰减。另一方面，碳具有还原性，在㷽烧过程 中可将痕量的 $\mathrm{Ti}^{4+}$ 还原为 $\mathrm{Ti}^{3+}$, 从而提高电子电导

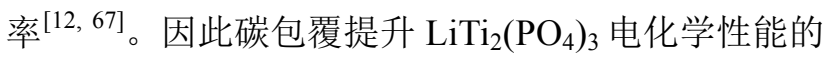
机制在于表面悬空键、石墨化碳层和痕量 $\mathrm{Ti}^{3+}$ 的共同 作用。

\subsection{2 碳源对 $\mathrm{LiTi}_{2}\left(\mathrm{PO}_{4}\right)_{3}$ 电化学性能的影响}

根据前驱体的种类，含碳试剂可分为有机碳源 和无机碳源。在高温裂解时, 有机碳源可形成同质化 碳层, 其碳层厚度易于控制, 但石墨化程度通常难 以控制, 无机碳源有助于形成 3D 导电网络结构, 但 对单一颗粒的表面包覆可能效果不佳, 因此同时引 入有机和无机碳源可以提供互补优势 ${ }^{[68]}$ 。有机碳源 主要包括柠檬酸、葡萄糖、蔗糖、淀粉和聚乙烯醇 等, 无机碳源主要有乙炔黑、石墨烯和碳纳米管 (Carbon nanotubes, CNTs)等。

(a)
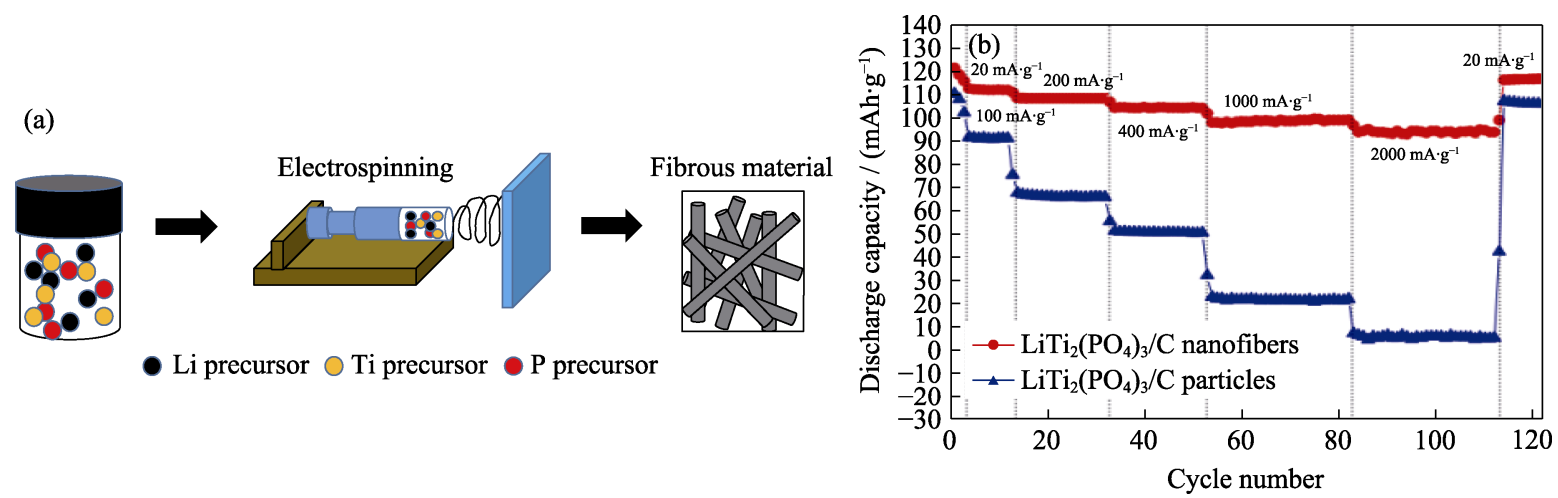

图 4 静电纺丝法示意图(a), $\mathrm{LiTi}_{2}\left(\mathrm{PO}_{4}\right)_{3}$ 纤维与颗粒的倍率性能曲线(b) ${ }^{[24]}$

Fig. 4 Schematic diagram of electrospinning (a), comparison of rate performance between $\mathrm{LiTi}_{2}\left(\mathrm{PO}_{4}\right)_{3}$ fibers and particles (b) ${ }^{[24]}$ 
叶嘉明等 ${ }^{[69]}$ 对比了葡萄糖、蔗糖、聚乙烯醇和 淀粉作为碳源对 $\mathrm{LiTi}_{2}\left(\mathrm{PO}_{4}\right)_{3}$ 电化学性能的影响。在 $0.1 C$ 倍率下经过 50 次循环后，采用葡萄糖为碳源制 备的 $\mathrm{LiTi}_{2}\left(\mathrm{PO}_{4}\right)_{3} / \mathrm{C}$ 复合负极材料的放电比容量为 $121.6 \mathrm{mAh} \cdot \mathrm{g}^{-1}$ ，明显高于蔗糖、聚乙烯醇和淀粉(69.8、 92.8 和 $\left.83.1 \mathrm{mAh} \cdot \mathrm{g}^{-1}\right)$ 。其原因可能在于几种碳源的 分子量和结构性质存在差异, 使得产物的碳化程 度、碳化历程和积碳含量有所不同。葡萄糖对电性 能的提高最为明显，其原因可能在于实现了晶化程 度和积碳含量的平衡。聚乙烯醇煅烧后的晶化程度 通常较高 ${ }^{[70]}$, 因而其电化学性能优于蔗糖和淀粉。蔗 糖包含环状结构，其裂解历程更为复杂，同时蔗糖

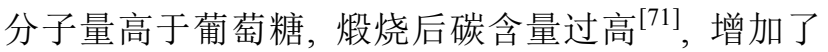
$\mathrm{Li}^{+}$脱嵌的阻力, 因而提升电化学性能的效果不佳。

张晨阳等 ${ }^{[72]}$ 以 $1 \mathrm{~mol} \cdot \mathrm{L}^{-1} \mathrm{Li}_{2} \mathrm{SO}_{4}$ 和 $2 \mathrm{~mol} \cdot \mathrm{L}^{-1}$ $\mathrm{ZnSO}_{4}$ 的混合溶液为电解液, 锌片为对电极和参比 电极组成扣式电池, 探讨了聚多巴胺、酚醛树脂、聚 丙烯腈和葡萄糖的包覆效果, 其倍率性能见图 5(a,b)。 酚醛树脂高温碳化后的结晶度更高, 导电性最佳,
其次为聚多巴胺，但聚多巴胺的附着力超强，可以 在多种纳米材料上形成均匀包覆层 ${ }^{[73]}$, 因而复合材 料的循环稳定性最佳, 在 $5 \mathrm{C}$ 倍率下, 聚多巴胺包覆 的 $\mathrm{LiTi}_{2}\left(\mathrm{PO}_{4}\right)_{3}$ 经 1000 次循环，容量保持率达 $86 \%$ 。

$\mathrm{He}$ 等 ${ }^{[74]}$ 以酚醛树脂为包覆碳源, 其水系全电池 $\mathrm{LiTi}_{2}\left(\mathrm{PO}_{4}\right)_{3} \mid$ 饱和 $\mathrm{Li}_{2} \mathrm{SO}_{4} \mid \mathrm{LiMn}_{2} \mathrm{O}_{4}$ 在 $1 C 、 10 C$ 和 $20 C$ 倍率下, 放电比容量分别为 $111.4 、 93.7 、 74.2 \mathrm{mAh} \cdot \mathrm{g}^{-1}$ 。 在 $5 C$ 倍率下循环 1000 次后，容量保持率为 $84.3 \%$ 。 Sun 等 ${ }^{[75]}$ 以酚醛树脂为碳源, 通过水热法合成了目 标材料。他们采用十二烷基苯磺酸钠对碳源和 $\mathrm{LiTi}_{2}\left(\mathrm{PO}_{4}\right)_{3}$ 前驱体进行分散, 降低了体系的表面能, 提高了碳源的分散程度, 使包覆层更加均匀。此外, 碳介孔有效缓解了颗粒在充放电过程中的体积膨胀, 增强了循环稳定性，机制如图 5(c)所示。其全电池 $\mathrm{LiTi}_{2}\left(\mathrm{PO}_{4}\right)_{3}\left|\mathrm{Li}_{2} \mathrm{SO}_{4}\right| \mathrm{LiMn}_{2} \mathrm{O}_{4}$ 在 $750 \mathrm{~mA} \cdot \mathrm{g}^{-1}$ 的电流密 度下经 5500 次循环, 容量保持率为 $82.7 \%$ 。

$\mathrm{Xu}$ 等 ${ }^{[76]}$ 在合成体系中引入了柠檬酸和 CNTs, 制备了 $\mathrm{LiTi}_{2}\left(\mathrm{PO}_{4}\right)_{3} @ \mathrm{C} / \mathrm{CNTs}$ 复合材料。柠檬酸在单 一 $\mathrm{LiTi}_{2}\left(\mathrm{PO}_{4}\right)_{3}$ 颗粒表面具有良好的包覆效果, CNTs
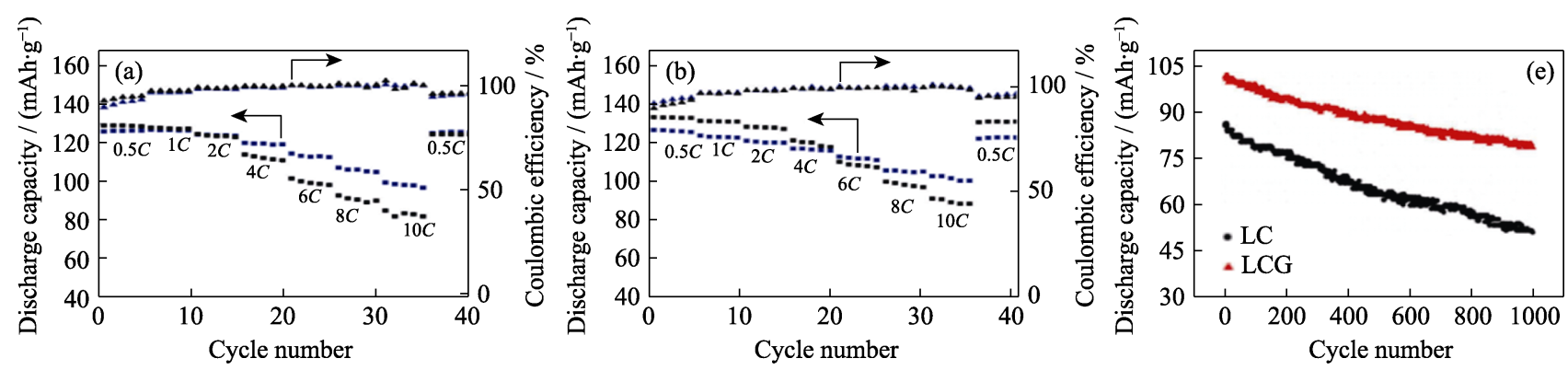

(c)

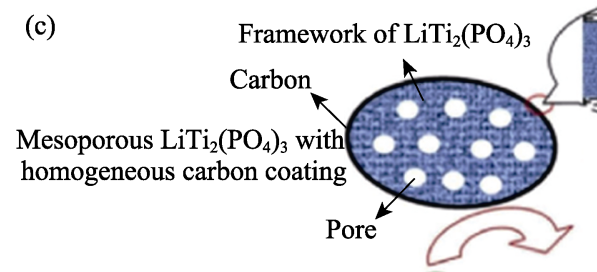

( $\mathrm{H}_{2} \mathrm{O}$
Li ion insertion process



Volume expansion during $\mathrm{Li}$ ion insertion could be well accommodated by the pores.

(d)

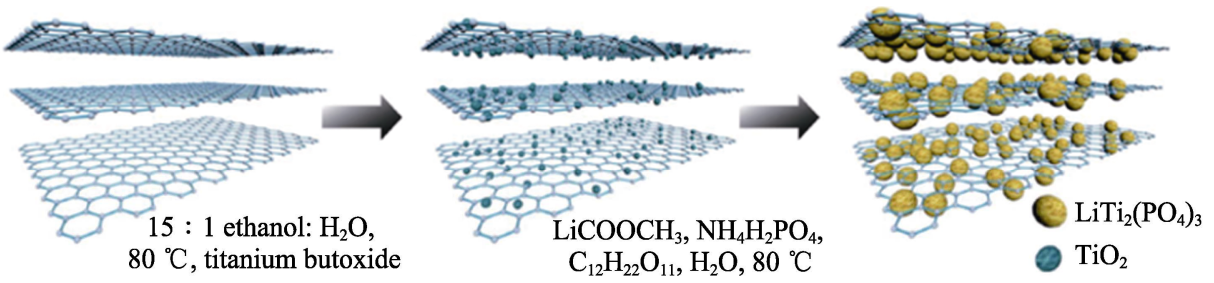

图 5 四种包覆碳源的倍率性能图 $(a, b)^{[72]}((a)$ 蓝、黑分别为聚多巴胺和酚醛树脂;

(b)蓝、黑分别为聚丙烯腈和葡萄糖), 具有均质碳层的介孔 $\mathrm{LiTi}_{2}\left(\mathrm{PO}_{4}\right)_{3}$ 中 $\mathrm{Li}^{+}$插入的机制示意图(c) ${ }^{[75]}$, rGO-LTP 的合成步骤示意图(d) ${ }^{[78]}, \mathrm{LC}$ 和 LCG 在 $5 C$ 下循环 1000 次的循环性能曲线(e) ${ }^{[80]}$

Fig. 5 Comparison chart of rate performance of four coated carbon sources ( $a, b)^{[72]}$ (blue and black in (a) indicating polydopamine and phenolic resin; blue and black in (b) indicating polyacrylonitrile and glucose), schematic illustration of the tentative $\mathrm{Li}^{+}$insertion mechanism in mesoporous $\operatorname{LiTi}_{2}\left(\mathrm{PO}_{4}\right)_{3}$ with carbon coating layer $(\mathrm{c})^{[75]}$, schematic diagram of the synthesis steps of rGO-LTP $(\mathrm{d})^{[78]}$, and cyclic performance of LC and LCG anodes at $5 C$ for 1000 cycles $(\mathrm{e})^{[80]}$ 
搭建了三维导电网络, 使 $\mathrm{LiTi}_{2}\left(\mathrm{PO}_{4}\right)_{3}$ 颗粒分散于其 中。与单一的柠檬酸包覆相比, 引入 CNTs 提高了材 料的电子电导率, 抑制了极化现象。其水系全电池 $\mathrm{LiTi}_{2}\left(\mathrm{PO}_{4}\right)_{3}\left|5 \mathrm{~mol} \cdot \mathrm{L}^{-1} \mathrm{LiNO}_{3}\right| \mathrm{LiMn}_{2} \mathrm{O}_{4}$ 在 $3 \mathrm{~A} \cdot \mathrm{g}^{-1}$ 的充 电倍率下，经过 500 次循环，容量保持率为 $72.9 \%$ 。

Roh 等 ${ }^{[77]}$ 将锂源、钛源、磷源与氧化石墨烯的 二乙二醇溶液混合, 在水热反应釜中进行反应, 获 得前驱体, 随后于 $800{ }^{\circ} \mathrm{C}$ 恒温 $10 \mathrm{~h}$ 获得复合材料, 探讨了其半电池的电化学性能。在 $1 C$ 倍率下, 经 100 次循环, 放电比容量为初始值的 $93.2 \%$, 库仑效 率接近 $100 \%$ 。 $\mathrm{Lim}$ 等 ${ }^{[78]}$ 在合成体系中引入蔗糖和氧 化石墨烯(GO)双碳源, 石墨烯可在 $\mathrm{LiTi}_{2}\left(\mathrm{PO}_{4}\right)_{3}$ 周围 形成导电网络, 而蔗糖包覆在单一 $\operatorname{LiTi}_{2}\left(\mathrm{PO}_{4}\right)_{3}$ 颗粒 表面, 两种碳源形成优势互补, 其合成思路如图 5(d) 所示。该课题组首先将 $\mathrm{Ti}\left(\mathrm{C}_{4} \mathrm{H}_{9} \mathrm{O}\right)_{4}$ 与氧化石墨烯置 于水-乙醇中 $80{ }^{\circ} \mathrm{C}$ 搅拌 $12 \mathrm{~h}$, 混合过程中, $\mathrm{Ti}\left(\mathrm{C}_{4} \mathrm{H}_{9} \mathrm{O}\right)_{4}$ 发生水解, 产物在氧化石墨烯表面生长, 得到 $\mathrm{GO}-\mathrm{TiO}_{2}$ 混合物, 随后加入锂源、磷源和蔗糖, 在惰性气氛下 $900{ }^{\circ} \mathrm{C}$ 恒温 $12 \mathrm{~h}$, 获得了具有导电网络 结构的复合材料(rGO-LTP)。该方法确保了 $\mathrm{LiTi}_{2}\left(\mathrm{PO}_{4}\right)_{3}$ 颗粒 $(200 \mathrm{~nm})$ 在氧化石墨烯表面原位生长, 理论上 防止了团聚。 $\mathrm{LiTi}_{2}\left(\mathrm{PO}_{4}\right)_{3}$ 通过 $\mathrm{Ti} / \mathrm{P}-\mathrm{O}-\mathrm{C}$ 键与氧化石 墨烯结合, 或通过范德瓦尔斯力与氧化石墨烯的芳 环区域结合 ${ }^{[79]}$ 。该复合材料的碳含量低至 $1.79 \%$, 在 未引入导电剂的情况下依然展现出了优异的电化学性 能。在 $210 \mathrm{Wh} \cdot \mathrm{kg}^{-1}$ 的能量密度下, 比功率为 $10000 \mathrm{~W} \cdot \mathrm{kg}^{-1}$ 。在 $10 C$ 倍率下, 100 次循环后, 容量保 持率为 $92 \%$ 。Zhou 等 ${ }^{[80]}$ 采用同样的策略, 以酚醛树
脂和氧化石墨烯为双碳源对 $\mathrm{LiTi}_{2}\left(\mathrm{PO}_{4}\right)_{3}$ 进行改性, 获得了 $\mathrm{LiTi}_{2}\left(\mathrm{PO}_{4}\right)_{3} / \mathrm{C} /$ 石墨烯复合材料(LCG), 其碳 的质量分数约为 $16.2 \%$ 。在 $5 C$ 倍率下, 全电池体系 $\mathrm{LiTi}_{2}\left(\mathrm{PO}_{4}\right)_{3}\left|\mathrm{Li}_{2} \mathrm{SO}_{4}\right| \mathrm{LiMn}_{2} \mathrm{O}_{4}$ 的初始放电比容量为 $101.1 \mathrm{mAh} \cdot \mathrm{g}^{-1}$ 。在 $5 C$ 倍率下循环 1000 次后, 与单碳 源包覆材料(LC)相比, 其容量保持率提升了 $19.1 \%$, 如图 5(e)所示。

\subsection{3 包覆方法对 $\mathrm{LiTi}_{2}\left(\mathrm{PO}_{4}\right)_{3}$ 电化学性能的影响}

根据包覆方式不同, 碳包覆可以分为原位和非 原位包覆。原位包覆即在原料体系中引入含碳试剂, 待各组分充分混合均匀后置于高温加热设备中进行 制烧。而非原位包覆则是将纯相 $\mathrm{LiTi}_{2}\left(\mathrm{PO}_{4}\right)_{3}$ 与碳源 进行混合并炦烧。表 4 对比了采用不同碳源及包覆 方式所得到的 $\mathrm{LiTi}_{2}\left(\mathrm{PO}_{4}\right)_{3}$ 的电化学性能参数。由表 可知, 非原位碳包覆对材料循环寿命的延长和容量 保持率的提升效果通常低于原位包覆, 其主要原因 在于含碳试剂分散性的差别。对于原位包覆而言, 在 前驱体内, 含碳试剂与原料的接触面积更大、分散更 均匀, 在煅烧过程中可以形成分散性更好的碳层, 对电性能的提高更明显。而非原位包覆则是基于固 相混料, 但固相混料的均匀度较差, 因此碳包覆更 多选择原位法。通过对以上结果的归纳可以初步判 断, 在 $\mathrm{LiTi}_{2}\left(\mathrm{PO}_{4}\right)_{3}$ 表面进行碳包覆可以减小颗粒尺 寸、阻止电解液与 $\mathrm{LiTi}_{2}\left(\mathrm{PO}_{4}\right)_{3}$ 的直接接触, 从而抑制 副反应, 最终提高产物的倍率性能和容量保持率。适 当的碳层厚度可以改善电子电导率, 较低的碳含量 对电子电导率的提升有限, 而当碳含量过高时则会 增加 $\mathrm{Li}^{+}$嵌入-脱出时的阻力, 不利于改善电性能。

表 4 溶胶一凝胶法不同碳源和包覆方式的电性能比较

Table 4 Comparison of electrochemical performance of different carbon sources and coating methods by Sol-Gel

\begin{tabular}{|c|c|c|c|c|c|c|c|}
\hline $\begin{array}{l}\text { Calcination } \\
\text { parameter }\end{array}$ & $\begin{array}{l}\text { Coating } \\
\text { method }\end{array}$ & Carbon source & $\begin{array}{l}\text { Mass fraction of } \\
\text { carbon } / \%\end{array}$ & $\begin{array}{c}\text { Current } \\
\text { density } /\left(\mathrm{mA}^{-1} \mathrm{~g}^{-1}\right)\end{array}$ & $\begin{array}{l}\text { Specific capacity } \\
(\text { cycles }) /\left(\mathrm{mAh} \cdot \mathrm{g}^{-1}\right)\end{array}$ & $\begin{array}{c}\text { Capacity } \\
\text { retention/\% }\end{array}$ & Ref. \\
\hline $800{ }^{\circ} \mathrm{C}-12 \mathrm{~h}$ & In-situ & Citric acid & 6.2 & 138 & $106.1(1)-89(1300)$ & 84 & [36] \\
\hline $900{ }^{\circ} \mathrm{C}-12 \mathrm{~h}$ & Ex-situ & Toluene & 12 & 700 & $100(1)-83(200)$ & 83 & {$[31]$} \\
\hline $800{ }^{\circ} \mathrm{C}-12 \mathrm{~h}$ & Ex-situ & Acetylene Black & 18 & 140 & $106.3(1)-86.5(100)$ & 81 & [81] \\
\hline $850{ }^{\circ} \mathrm{C}-12 \mathrm{~h}$ & Ex-situ & Acetylene Black & - & 1400 & $91.3(1)-74.4(100)$ & 81 & {$[82]$} \\
\hline $700{ }^{\circ} \mathrm{C}-12 \mathrm{~h}$ & In-situ & Pitch & 17.5 & 1380 & $107(1)-75.5(1000)$ & 70 & {$[83]$} \\
\hline $550{ }^{\circ} \mathrm{C}-24 \mathrm{~h}$ & In-situ & Sucrose & 3.5 & 1400 & $110(1)-104(800)$ & 94 & {$[17]$} \\
\hline $750{ }^{\circ} \mathrm{C}-5 \mathrm{~h}$ & In-situ & Polyaniline & 5.9 & 276 & 115.2(1)-94.6(1000) & 82 & {$[84]$} \\
\hline $750{ }^{\circ} \mathrm{C}-5 \mathrm{~h}$ & In-situ & Polyacrylonitrile & 5.9 & 690 & $95(1)-82.1(1000)$ & 86 & {$[85]$} \\
\hline $900{ }^{\circ} \mathrm{C}-12 \mathrm{~h}$ & In-situ & Graphene oxide & 1.79 & $\sim 1380$ & $110(1)-100(100)$ & 91 & {$[78]$} \\
\hline $800{ }^{\circ} \mathrm{C}-10 \mathrm{~h}$ & In-situ & Graphene oxide & - & $\sim 276$ & $105(1)-97.86(100)$ & 93.2 & [77] \\
\hline $700{ }^{\circ} \mathrm{C}-5 \mathrm{~h}$ & In-situ & $\begin{array}{l}\text { Graphene oxide, } \\
\text { phenolic resin }\end{array}$ & 16.2 & $\sim 690$ & $101.1(1)-78(1000)$ & 77.2 & {$[80]$} \\
\hline $800{ }^{\circ} \mathrm{C}-8 \mathrm{~h}$ & Ex-situ & $\beta$-Cyclodextrin & 3.13 & $\sim 690$ & $120(1)-111.3(200)$ & 88.7 & {$[86]$} \\
\hline
\end{tabular}




\section{3 元素掺杂}

元素掺杂, 即在晶格内部引入一种或多种元素。 根据掺杂元素的种类，可分为金属元素掺杂和非金 属元素掺杂。金属元素掺杂占据晶格中的 $\mathrm{Li}$ 或 $\mathrm{Ti}$ 位。目前, 常见的金属掺杂元素主要有 $\mathrm{Sn} 、 \mathrm{~K} 、 \mathrm{Na}$ 等。非金属元素占据 $\mathrm{LiTi}_{2}\left(\mathrm{PO}_{4}\right)_{3}$ 晶格中的阴离子位。 见诸报道的非金属掺杂元素较少，仅有卤族元素。元 素掺杂对电性能的提升主要归因于掺杂元素降低了 电荷转移阻抗 $R_{\mathrm{ct}}$, 并拓宽了晶格中 $\mathrm{Li}^{+}$的传输通道, 导致 $\mathrm{Li}^{+}$扩散系数增大。

\subsection{1 金属元素掺杂}

Bounar ${ }^{[87]} 、 \mathrm{Liu}^{[24]}$ 和 $\mathrm{He}$ 等 ${ }^{[88]}$ 分别在 $\mathrm{LiTi}_{2}\left(\mathrm{PO}_{4}\right)_{3}$ 晶格中掺杂 $\mathrm{Sn}$ 元素，但由于合成方法和原料不同， 最佳掺杂量和产物电化学性能也有所差别。Bounar 等 ${ }^{[87]}$ 主要探究了 $\mathrm{Sn}$ 元素对晶体结构的影响, 认为在 晶格中, $\mathrm{Ti}$ 与 $\mathrm{Sn}$ 的最佳摩尔比为 $7: 3$, 相应的离子电 导率为 $2.17 \times 10^{-4} \mathrm{~S} \cdot \mathrm{cm}^{-1}$, 较掺杂前提升了两个数量 级, 适量的掺杂仅引起晶胞参数的线性变化, 而空 间群的种类并未发生改变。

Liu 等 ${ }^{[24]}$ 的合成产物兼具优良的倍率性能和循 环寿命。他们采用静电纺丝法制备了 $\mathrm{LiTi}_{1.8} \mathrm{Sn}_{0.2}\left(\mathrm{PO}_{4}\right)_{3} / \mathrm{C}$ 复合材料, 掺杂后该复合材料展现出非常优异的大 电流充放电性能, 掺杂前后的电化学性能对比如图 6 所示。在 $20 \mathrm{~mA} \cdot \mathrm{g}^{-1}$ 电流密度下, 初始放电比容量为 $116.2 \mathrm{mAh} \cdot \mathrm{g}^{-1}$ 。在电流密度为 $1000 、 2000 、 4000$ 、 $6000 \mathrm{~mA} \cdot \mathrm{g}^{-1}$ 的测试条件下，比容量分别为 $104 、 102$ 、 100、94 $\mathrm{mAh} \cdot \mathrm{g}^{-1}$ 。在 $600 \mathrm{C}$ 充电倍率下, 比容量为 $68.9 \mathrm{mAh} \cdot \mathrm{g}^{-1}$, 这是目前为止文献记录的最高充电倍 率。 $\mathrm{He}$ 等 ${ }^{[88]}$ 以 $\mathrm{Sn}$ 为掺杂元素, 选择了能耗较低、工 艺相对简单的溶胶一凝胶法, 获得了颗粒状材料 $\mathrm{LiTi}_{1.7} \mathrm{Sn}_{0.3}\left(\mathrm{PO}_{4}\right)_{3} / \mathrm{C}$ 。其水系锂离子全电池
$\mathrm{LiTi}_{1.7} \mathrm{Sn}_{0.3}\left(\mathrm{PO}_{4}\right)_{3} / \mathrm{C}\left|\mathrm{Li}_{2} \mathrm{SO}_{4}\right| \mathrm{LiMn}_{2} \mathrm{O}_{4}$ 展现出优异的 循环寿命及倍率性能。在 $0.2 C 、 6 C$ 和 $20 C$ 倍率下，放 电比容量分别为 $118.9 、 115.3 、 104.2 \mathrm{mAh} \cdot \mathrm{g}^{-1}$ 。即 使在 $10 C$ 倍率下, 1000 次循环后，其容量保持率仍可 达 $76.8 \%$ 。

$\mathrm{Na}$ 与 $\mathrm{K}$ 为同族元素, 占据晶格中的 $\mathrm{Li}$ 位。 $\mathrm{Na}$ 和 $\mathrm{K}$ 元素对电化学性能的提升主要归因于其增大了 晶格体积, 拓宽了 $\mathrm{Li}^{+}$传输通道。 $\mathrm{Liu}$ 等 ${ }^{[89]}$ 采用溶胶一 凝胶法制备了 $\mathrm{Li}_{0.97} \mathrm{Na}_{0.03} \mathrm{Ti}_{2}\left(\mathrm{PO}_{4}\right)_{3} / \mathrm{C}$ 复合材料。掺 杂后, $\mathrm{Li}^{+}$的扩散系数由 $1.6 \times 10^{-8} \mathrm{~cm}^{2} \cdot \mathrm{s}^{-1}$ 提升至 $8.9 \times 10^{-8} \mathrm{~cm}^{2} \cdot \mathrm{s}^{-1}$ 。Jiang 等 ${ }^{[21]}$ 采用相同的制备方法合 成了 $\mathrm{K}$ 元素掺杂的 $\mathrm{Li}_{1-x} \mathrm{~K}_{x} \mathrm{Ti}_{2}\left(\mathrm{PO}_{4}\right)_{3} / \mathrm{C}$ 纳米复合材料, 当 $x=0.03$ 时电化学性能最佳, $0.2 C$ 倍率下的初始放 电比容量为 $104.4 \mathrm{mAh} \cdot \mathrm{g}^{-1}$, 在 $6 \mathrm{C}$ 倍率下, 1000 次循 环后，容量保持率为 $78.8 \%$ 。

\subsection{2 非金属元素掺杂}

目前报道的非金属元素掺杂仅有卤族元素，它 们占据 $\mathrm{LiTi}_{2}\left(\mathrm{PO}_{4}\right)_{3}$ 的阴离子位。Wang 和 Zhang 等 ${ }^{[90-91]}$ 通过溶胶-凝胶法合成了 $\mathrm{F}$ 掺杂碳包覆的 $\mathrm{LiTi}_{2}\left(\mathrm{PO}_{4}\right)_{3-x} \mathrm{~F}_{x}$ 电极材料, 其粒径为 $20 \sim 50 \mathrm{~nm}$, 碳层 厚度为 $5 \mathrm{~nm}$, 最佳掺杂量为 $x=0.12$ 。其理论计算结 果表明，掺杂后禁带宽度由 $2.504 \mathrm{eV}$ 降低至 $1.931 \mathrm{eV，}$ 表明掺杂后材料的本征电子电导率得到了提高。其水 系全电池 $\mathrm{LiTi}_{2}\left(\mathrm{PO}_{4}\right)_{0.88} \mathrm{~F}_{0.12}\left|2 \mathrm{~mol} \cdot \mathrm{L}^{-1} \quad \mathrm{Li}_{2} \mathrm{SO}_{4}\right| \mathrm{LiMn}_{2} \mathrm{O}_{4}$ 的功率密度和能量密度达到了 2794.2 和 $43.7 \mathrm{Wh} \cdot \mathrm{kg}^{-1}$ 。 受到 Wang 等研究的启发, Luo 等 ${ }^{[92]}$ 在晶格中掺杂了 氯元素。掺杂后，在 $5 C$ 倍率下, 1000 次循环后，其容 量保持率由掺杂前的 $35.3 \%$ 升至 $61.3 \%$ 。 Jiang 等 ${ }^{[93]}$ 对 $\mathrm{LiTi}_{2}\left(\mathrm{PO}_{4}\right)_{3}$ 进行澳掺杂和碳包覆, 得到的 $\mathrm{LiTi}_{2}\left(\mathrm{PO}_{4}\right)_{2.82} \mathrm{Br}_{0.18} / \mathrm{C}$ 复合材料在 $15 \mathrm{C}$ 倍率下的初始 放电比容量达到 $86.3 \mathrm{mAh} \cdot \mathrm{g}^{-1}$ 。
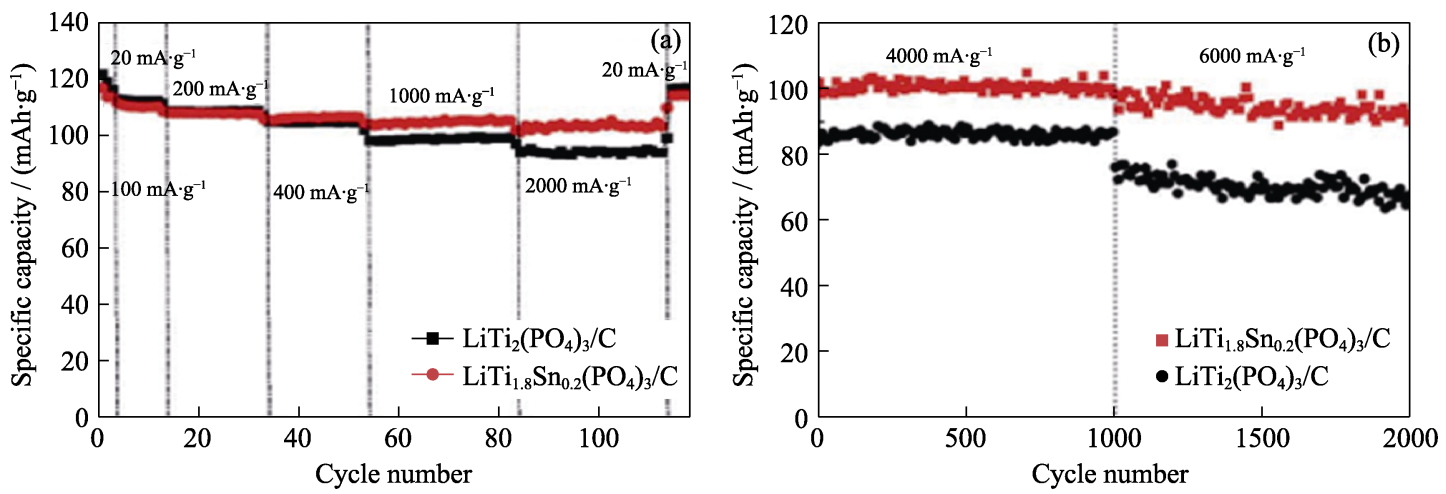

图 $6 \mathrm{LiTi}_{2}\left(\mathrm{PO}_{4}\right)_{3} / \mathrm{C}$ 和 $\mathrm{LiTi}_{1.8} \mathrm{Sn}_{0.2}\left(\mathrm{PO}_{4}\right)_{3} / \mathrm{C}$ 在不同电流密度下 连续循环的放电容量 (a), 电流密度为 4 和 $6 \mathrm{~A} \cdot \mathrm{g}^{-1}$ 时的长期循环性能( $(\mathrm{b})^{[24]}$

Fig. 6 Discharge capacity for successive cycling at different current densities (a), long-term cycling behavior at current densities of 4 and $6 \mathrm{~A} \cdot \mathrm{g}^{-1}(\mathrm{~b})$ of $\mathrm{LiTi}_{2}\left(\mathrm{PO}_{4}\right)_{3} / \mathrm{C}$ and $\mathrm{LiTi}_{1.8} \mathrm{Sn}_{0.2}\left(\mathrm{PO}_{4}\right)_{3} / \mathrm{C}^{[24]}$ 


\section{4 结束语}

$\mathrm{LiTi}_{2}\left(\mathrm{PO}_{4}\right)_{3}$ 具有合适的嵌锂电位和稳定的骨架 结构, 是一种有发展前景的水系锂离子电池负极用 活性材料, 其主要合成方法有高温固相法、共沉淀 法、溶胶-凝胶法和水/溶剂热法等。高温固相法最适 宜工业化, 但产物颗粒较大, 电化学性能较差。当前 的固相法主要以 $\mathrm{NH}_{4} \mathrm{H}_{2} \mathrm{PO}_{4}$ 为磷源, 高温时会产生 $\mathrm{NH}_{3}$, 腐蚀设备并污染环境。因此, 亟待开发绿色的 新型工艺, 以解决当前存在的问题。共沉淀法的产物 粒径小, 电化学性能优异, 同时具有易于工业化的 优势。但目前关于该工艺路线的探究较少, 且主要以 $\mathrm{Ti}\left(\mathrm{C}_{4} \mathrm{H}_{9} \mathrm{O}\right)_{4}$ 为钛源, 该原料极易水解变质, 增加了工 业化成本, 因而亟待开发以稳定的无机钛盐为原料 的新工艺。溶胶一凝胶法是实验室最常用的合成方法 之一，其㷽烧温度低、时间短，但工业化难度稍大。 水/溶剂热法可以获得具有一定特殊形貌的颗粒, 产 物的电化学性能通常较好。水体系电解液环境通常 较为复杂, 为使 $\mathrm{LiTi}_{2}\left(\mathrm{PO}_{4}\right)_{3}$ 更好地适应水系电解液, $\mathrm{LiTi}_{2}\left(\mathrm{PO}_{4}\right)_{3}$ 需要进行改性方能更明显地展现自身优 势。目前, 主要的思路有: 碳包覆、元素掺杂、颗粒 纳米化及形貌控制。在目前的碳包覆研究中, 研究人 员多以有机物为碳源, 但有机试剂优势单一, 若能 在体系中同时引入有机和无机碳源，将会实现更优 异的包覆效果, 大大提高电化学性能。元素掺杂可以 提高材料的本征电子电导率和离子扩散系数, 其作 用机理及对嵌锂行为的影响值得深入探究。这些改 性方法各具特色，若单独使用，则改善效果有限，因 此, 彼此间需要进行有机结合。理论计算作为一种较 先进的研究手段, 未来可为 $\mathrm{LiTi}_{2}\left(\mathrm{PO}_{4}\right)_{3}$ 的掺杂改性 和动力学研究提供理论指导。其中, 密度泛函理论可 以计算 $\mathrm{LiTi}_{2}\left(\mathrm{PO}_{4}\right)_{3}$ 晶面生长的表面热力学稳定性和 电子结构、探索各晶面的生长速率、分析改性机理、 预测最佳反应条件、优选掺杂元素等。但理论计算 并不能完全替代实验, 应当进一步研究如何实现两 者的有机结合。

\section{参考文献:}

[1] WENG Y, XU S, HUANG G, et al. Synthesis and performance of $\mathrm{Li}\left[\left(\mathrm{Ni}_{1 / 3} \mathrm{Co}_{1 / 3} \mathrm{Mn}_{1 / 3}\right)_{(1-x)} \mathrm{Mg}_{x}\right] \mathrm{O}_{2}$ prepared from spent lithium ion batteries. Jounral of Hazard Materials, 2013, 246-247: 163-172.

[2] KIM T, SONG W, SON D Y, et al. Lithium-ion batteries: outlook on present, future, and hybridized technologies. Journal of Materials Chemistry A, 2019, 7(7): 2942-2964.

[3] ARMAND M, TARASCON J M. Building better batteries. Nature, 2008, 451: 652-657.
[4] ZHANG $\mathrm{H}, \mathrm{ZHAO} \mathrm{H}, \mathrm{KHAN} \mathrm{M}$ A, et al. Recent progress in advanced electrode materials, separators and electrolytes for lithium batteries. Journal of Materials Chemistry A, 2018, 6(42): 20564-20620.

[5] GOODENOUGH J B, PARK K S. The Li-ion rechargeable battery: a perspective. Journal of the American Chemical Society, 2013, 135(4): 1167-1176.

[6] SUO L, L H. The past, present and future of lithium ion batteries. Physics, 2020, 49(1): 17-23.

[7] LI W, DAHN J R, WAINWRIGHT D S. Rechargeable lithium batteries with aqueous electrolytes. Science, 1994, 264(5162): 1115-1118.

[8] ZHOU D. A New Anode Material of $\mathrm{Na}_{2} \mathrm{~V}_{6} \mathrm{O}_{16}$ Nanowires for Aqueous Rechargeable Lithium Battery. Changsha: Central South University, Master Dissertation, 2013.

[9] LI W, MCKINNON W R, R D J. Lithium intercalation from aqueous solutions. Journal of Electrochemical Society, 1994, 141: 2310-2316.

[10] TANG W, ZHU Y, HOU Y, et al. Aqueous rechargeable lithium batteries as an energy storage system of superfast charging. Energy \& Environmental Science, 2013, 6(7): 2093-2104.

[11] DEMIR-CAKAN R, PALACIN M R, CROGUENNEC L. Rechargeable aqueous electrolyte batteries: from univalent to multivalent cation chemistry. Journal of Materials Chemistry A, 2019, 7(36): 20519-20539.

[12] LUO J Y, CUI W J, HE P, et al. Raising the cycling stability of aqueous lithium-ion batteries by eliminating oxygen in the electrolyte. Nature Chemistry, 2010, 2(9): 760-765.

[13] LIU Z, HUANG Y, HUANG Y, et al. Voltage issue of aqueous rechargeable metal-ion batteries. Chemical Society Review, 2020, 49(1): 180-232.

[14] LIU W, WANG B, LI L. Recent progress in electrode materials for aqueous lithium-ion batteries. Energy Storage Science and Technology, 2014, 3(1): 9-20.

[15] AATIQ A, MENETRIER M, CROGUENNEC L, et al. On the structure of $\mathrm{Li}_{3} \mathrm{Ti}_{2}\left(\mathrm{PO}_{4}\right)_{3}$. Journal of Materials Chemistry, 2002, 12(10): 2971-2978.

[16] GIAROLA M, SANSON A, TIETZ F, et al. Structure and vibrational dynamics of nasicon-type $\operatorname{LiTi}_{2}\left(\mathrm{PO}_{4}\right)_{3}$. Journal of Physical Chemistry C, 2017, 121(7): 3697-3706.

[17] EL-SHINAWI H, JANEK J. Low-temperature synthesis of macroporous $\mathrm{LiTi}_{2}\left(\mathrm{PO}_{4}\right)_{3} / \mathrm{C}$ with superior lithium storage properties. RSC Advances, 2015, 5(19): 14887-14891.

[18] GUTIERREZ A, BENEDEK N A, MANTHIRAM A. Crystalchemical guide for understanding redox energy variations of $\mathrm{M}^{2+} \beta^{+}$ couples in polyanion cathodes for lithium-ion batteries. Chemistry of Materials, 2013, 25(20): 4010-4016.

[19] DELMAS C, NADIRI A, SOUBEYROUX L J. The nasicon-type titatium phosphates $\mathrm{ATi}_{2}\left(\mathrm{PO}_{4}\right)_{3}(\mathrm{~A}=\mathrm{Li}, \mathrm{Na})$ as electrode materials. Solid State Ionics, 1988, 28-30: 419-423.

[20] WANG H, HUANG K, ZENG Y, et al. Electrochemical properties of $\mathrm{TiP}_{2} \mathrm{O}_{7}$ and $\mathrm{LiTi}_{2}\left(\mathrm{PO}_{4}\right)_{3}$ as anode material for lithium ion battery with aqueous solution electrolyte. Electrochimica Acta, 2007, 52(9): 3280-3285.

[21] JIANG Z, LI Y, HAN C, et al. $\mathrm{K}$ doping on Li site enables $\mathrm{LiTi}_{2}\left(\mathrm{PO}_{4}\right)_{3} / \mathrm{C}$ excellent lithium storage performance. Solid State Ionics, 2019, 341: 115036.

[22] YU S, TEMPEL H, SCHIERHOLZ R, et al. $\mathrm{LiTi}_{2}\left(\mathrm{PO}_{4}\right)_{3} / \mathrm{C}$ anode material with a spindle-like morphology for batteries with high rate capability and improved cycle life. ChemElectroChem, 2016, 3(7): 1157-1169.

[23] SUN J, SUN Y, GAI L, et al. Carbon-coated mesoporous $\mathrm{LiTi}_{2}\left(\mathrm{PO}_{4}\right)_{3}$ nanocrystals with superior performance for lithium-ion 
batteries. Electrochimica Acta, 2016, 200: 66-74.

[24] LIU L, SONG T, HAN H, et al. Electrospun Sn-doped $\mathrm{LiTi}_{2}\left(\mathrm{PO}_{4}\right)_{3} / \mathrm{C}$ nanofibers for ultra-fast charging and discharging. Journal of Materials Chemistry A, 2015, 3(19): 10395-10402.

[25] WANG G X, BRADHURST D H, DOU S X, et al. $\operatorname{LiTi}_{2}\left(\mathrm{PO}_{4}\right)_{3}$ with NASICON-type structure as lithium-storage materials. Journal of Power Sources, 2003, 124(1): 231-236.

[26] LI W, DAHN J R. Lithium-ion cells with aqueous electrolytes. Journal of Electrochemical Society, 1995, 142: 1742-1746.

[27] KOHLER J, MAKIHARA H, UEGAITO $\mathrm{H}$, et al. $\mathrm{LiV}_{3} \mathrm{O}_{8}$ : characterization as anode material for an aqueous rechargeable Li-ion battery system. Electrochim. Acta, 2000, 46: 59-65.

[28] ZHENG W. Solid-state Synthesis and Surface Modification of $\mathrm{LiFePO}_{4}$ and $\mathrm{LiTi}_{2}\left(\mathrm{PO}_{4}\right)_{3}$ for Lithium Ion Electrode Materials. Zhengjiang: Zhengjiang University, Doctoral Dissertation, 2010.

[29] FENG C, LI L, TANG J, et al. Synthesis and electrochemical performance of a new type of anode material $\mathrm{LiTi}_{2}\left(\mathrm{PO}_{4}\right)_{3}$. Power Technology, 2015, 39(2): 242-244.

[30] LI W, LI Y, CAO M, et al. Synthesis and electrochemical performance of alginic acid-based carbon-coated $\mathrm{Li}_{3} \mathrm{~V}_{2}\left(\mathrm{PO}_{4}\right)_{3}$ composite by rheological phase method. Acta Phys-ChimSin, 2017, 33(11): 2261-2267.

[31] LUO J Y, XIA Y Y. Aqueous lithium-ion battery $\mathrm{LiTi}_{2}\left(\mathrm{PO}_{4}\right)_{3} / \mathrm{LiMn}_{2} \mathrm{O}_{4}$ with high power and energy densities as well as superior cycling stability. Advanced Functional Materials, 2007, 17(18): 3877-3884.

[32] TANG Z K, XUE Y F, TEOBALDI G, et al. The oxygen vacancy in Li-ion battery cathode materials. Nanoscale Horizons, 2020, 5(11): 1453-1466.

[33] LUO J Y, CHEN L J, ZHAO Y J, et al. The effect of oxygen vacancies on the structure and electrochemistry of $\mathrm{LiTi}_{2}\left(\mathrm{PO}_{4}\right)_{3}$ for lithium-ion batteries: a combined experimental and theoretical study. Journal of Power Sources, 2009, 194(2): 1075-1080.

[34] CHENG C. Study of Anode Materials for Aqueous Rechargeable Lithium-ion Batteries. Changsha: Xiangtan University, Master Dissertation, 2010.

[35] MARIAPPAN C R, GALVEN C, CROSNIER-LOPEZ M P, et al. Synthesis of nanostructured $\mathrm{LiTi}_{2}\left(\mathrm{PO}_{4}\right)_{3}$ powder by a Pechini-type polymerizable complex method. Journal of Solid State Chemistry, 2006, 179(2): 450-456.

[36] WESSELLS C, HUGGINS R A, CUI Y. Recent results on aqueous electrolyte cells. Journal of Power Sources, 2011, 196(5): 2884-2888.

[37] ZHOU X L, YAN Z G, LI S Y, et al. Single crystalline $\operatorname{LiTi}_{2}\left(\mathrm{PO}_{4}\right)_{3}$ nanowires by porous template with improved electrochemical performance. Materials Today Energy, 2018, 7: 113-121.

[38] ZHOU X. Lithium Titanium Phosphate and Carbon/copper Composite Electrode Materials: Controlled Preparation, Structural Study and Electrochemical Performance. Beijing: Beijing University of Technology, Doctoral Dissertation, 2014.

[39] ZHOU D, LI J, CHEN C, et al. A hydrothermal synthesis of $\mathrm{Ru}$-doped $\mathrm{LiMn}_{1.5} \mathrm{Ni}_{0.5} \mathrm{O}_{4}$ cathode materials for enhanced electrochemical performance. RSC Advances, 2021, 11(21): 12549-12558.

[40] SONG Y, XIE B, SONG S, et al. Regeneration of $\mathrm{LiFePO}_{4}$ from spent lithium-ion batteries via a facile process featuring acid leaching and hydrothermal synthesis. Green Chemistry, 2021, 23(11): 3963-3971.

[41] WANG J, QIN X, GUO J, et al. A porous hierarchical micro/nano $\mathrm{LiNi}_{0.5} \mathrm{Mn}_{1.5} \mathrm{O}_{4}$ cathode material for $\mathrm{Li}$-ion batteries synthesized by a urea-assisted hydrothermal method. Dalton Transactions, 2018, 47(21): 7333-7343.

[42] QIN X, ZHOU M, ZONG B, et al. Urea-assisted hydrothermal synthesis of a hollow hierarchical $\mathrm{LiNi}_{0.5} \mathrm{Mn}_{1.5} \mathrm{O}_{4}$ cathode material with tunable morphology characteristics. RSC Advances, 2018,
8(53): 30087-30097.

[43] YUE Y, PANG W. Hydrothermal synthesis and characterization of $\mathrm{LiTi}_{2}\left(\mathrm{PO}_{4}\right)_{3}$. Journal of Materials Science Letters, 1990, 9: 1392.

[44] LIANG Y, HISAMO T, SUMI S, et al. Direct fabrication of thin-film $\mathrm{LiTi}_{2}\left(\mathrm{PO}_{4}\right)_{3}$ electrodes using the hydrothermal method. Solid State Ionics, 2016, 296: 7-12.

[45] LI M, LIU L, ZHANG N, et al. Mesoporous $\mathrm{LiTi}_{2}\left(\mathrm{PO}_{4}\right)_{3} / \mathrm{C}$ composite with trace amount of carbon as high-performance electrode materials for lithium ion batteries. Journal of Alloys and Compounds, 2018, 749: 1019-1027.

[46] HOU P, ZHANG H, ZI Z, et al. Core-shell and concentrationgradient cathodes prepared via co-precipitation reaction for advanced lithium-ion batteries. Journal of Materials Chemistry A, 2017, 5(9): 4254-4279.

[47] LI H, LI Z, CUI Y, et al. Long-cycled $\mathrm{Li}_{2} \mathrm{ZnTi}_{3} \mathrm{O}_{8} / \mathrm{TiO}_{2}$ composite anode material synthesized via a one-pot co-precipitation method for lithium ion batteries. New Journal of Chemistry, 2017, 41(3): 975-981.

[48] 杨勇. 固态电化学. 北京: 化学工业出版社, 2017.

[49] 盖利刚, 孙家香, 姜海辉. 一种碳包覆介孔磷酸钛锂的制备方 法: ZL201510957301.8. 2015.12.18.

[50] OGHBAEI M, MIRZAEE O. Microwave versus conventional sintering: a review of fundamentals, advantages and applications. Journal of Alloys and Compounds, 2010, 494(1/2): 175-189.

[51] RIQUET G, MARINEL S, BREARD Y, et al. Direct and hybrid microwave solid state synthesis of $\mathrm{CaCu}_{3} \mathrm{Ti}_{4} \mathrm{O}_{12}$ ceramic: microstructures and dielectric properties. Ceramics International, 2018, 44(13): 15228-15235.

[52] ZHANG M, GARCIA-ARAEZ N, HECTOR A L. Understanding and development of olivine $\mathrm{LiCoPO}_{4}$ cathode materials for lithium-ion batteries. Journal of Materials Chemistry A, 2018, 6(30): 14483-14517.

[53] LUDWIG J, NORDLUND D, DOEFF M M, et al. Synthesis and characterization of metastable, $20 \mathrm{~nm}$-sized $\mathrm{Pna}_{1}-\mathrm{LiCoPO}_{4}$ nanospheres. Journal of Solid State Chemistry, 2017, 248: 9-17.

[54] GUO X, JIA X, HU H, et al. Synthesis of $\mathrm{LiTi}_{2}\left(\mathrm{PO}_{4}\right)_{3}$ ultrafine powder by Sol-Gel and microwave heating method. Materials Reports, 2007, 21(11A): 68-71.

[55] HU J, HUANG W, YANG L, et al. Structure and performance of the $\mathrm{LiFePO}_{4}$ cathode material: from the bulk to the surface. Nanoscale, 2020, 12(28): 15036-15044.

[56] YANG C, LEE D J, KIM H, et al. Synthesis of nano-sized urchin-shaped $\mathrm{LiFePO}_{4}$ for lithium ion batteries. RSC Advances, 2019, 9(24): 13714-13721.

[57] XIANG J, ZHANG P, LV S, et al. Spinel $\mathrm{LiMn}_{2} \mathrm{O}_{4}$ nanoparticles fabricated by the flexible soft template/Pichini method as cathode materials for aqueous lithium-ion capacitors with high energy and power density. RSC Advances, 2021, 11(25): 14891-14898.

[58] JO J, NAM S, HAN S, et al. One-pot pyro synthesis of a nanosized- $\mathrm{LiMn}_{2} \mathrm{O}_{4} / \mathrm{C}$ cathode with enhanced lithium storage properties. RSC Advances, 2019, 9(42): 24030-24038.

[59] QI W, SHAPTER J G, WU Q, et al. Nanostructured anode materials for lithium-ion batteries: principle, recent progress and future perspectives. Journal of Materials Chemistry A, 2017, 5(37): 19521-19540.

[60] TIAN L, YU H, ZHANG W, et al. The star material of lithium ion batteries, $\mathrm{LiFePO}_{4}$ : basic properties, optimize moderation and future prospects. Materials Reports, 2019, 33(11): 3561-3579.

[61] DENG W, WANG X, LIU C, et al. Touching the theoretical capacity: synthesizing cubic $\mathrm{LiTi}_{2}\left(\mathrm{PO}_{4}\right)_{3} / \mathrm{C}$ nanocomposites for high-performance lithium-ion battery. Nanoscale, 2018, 10(14): 6282-6287.

[62] WU Y, CHONG S, LIU Y, et al. High electrochemical performance 
of nanocrystallized carbon-coated $\mathrm{LiFePO}_{4}$ modified by tris (pentafluorophenyl) borane as a cathode material for lithium-ion batteries. RSC Advances, 2018, 8(51): 28978-28986.

[63] WANG Y, WANG X, JIANG A, et al. A versatile nitrogen-doped carbon coating strategy to improve the electrochemical performance of $\mathrm{LiFePO}_{4}$ cathodes for lithium-ion batteries. Journal of Alloys and Compounds, 2019, 810: 151889.

[64] PARK G D, HONG J H, JUNG D S, et al. Unique structured microspheres with multishells comprising graphitic carbon-coated $\mathrm{Fe}_{3} \mathrm{O}_{4}$ hollow nanopowders as anode materials for high-performance Li-ion batteries. Journal of Materials Chemistry A, 2019, 7(26): 15766-15773.

[65] KU D J, LEE J H, LEE S J, et al. Effects of carbon coating on $\mathrm{LiNi}_{0.5} \mathrm{Mn}_{1.5} \mathrm{O}_{4}$ cathode material for lithium ion batteries using an atmospheric microwave plasma torch. Surface and Coatings Technology, 2019, 376: 25-30.

[66] SUN W, LIU J, LIU X, et al. Bimolecular-induced hierarchical nanoporous $\mathrm{LiTi}_{2}\left(\mathrm{PO}_{4}\right)_{3} / \mathrm{C}$ with superior high-rate and cycling performance. Chemical Communications, 2017, 53(62): 8703-8706.

[67] TAN Y, XUE B. Research progress on lithium titanate as anode material in lithium-ion battery. Journal of Inorganic Materials, 2018, 33(5): 475-482.

[68] LI H, ZHOU H. Enhancing the performances of Li-ion batteries by carbon-coating: present and future. Chemical Communications, 2012, 48(9): 1201-1217.

[69] YE J, LI C, RAO M, et al. Effects of different carbon solutions on electrochemical performance of $\mathrm{LiTi}_{2}\left(\mathrm{PO}_{4}\right)_{3} / \mathrm{C}$ composite anode material. Power Technology, 2020, 44(3): 322-325.

[70] LUO S, TIAN Y, TANG Z, et al. Effect of the structure of pyrolytic carbon on the performance of $\mathrm{LiFePO}_{4} / \mathrm{C}$ composite cathode material. Rare Metal Materials and Engineering, 2009, 38: 13-15.

[71] CHEN Y, HE H, LIU L, et al. Thermal decomposition of glucose and sucrose by kinetics analysis. The Chinese Joumal of Process Engineering, 2010, 10(4): 720-725.

[72] ZHANG C, WEN Y, ZHANG P, et al. Effect of organic carbon source on performance of $\mathrm{LiTi}_{2}\left(\mathrm{PO}_{4}\right)_{3} / \mathrm{C}$ composite electrodes in aqueous solutions. Chemical Journal of Chinese Universities, 2020, 41(6): 1352-1361.

[73] LIN L, CONG Z, CAO J, et al. Multifunctional $\mathrm{Fe}_{3} \mathrm{O}_{4} @$ polydopamine core-shell nanocomposites for intracellular mRNA detection and imaging-guided photothermal therapy. ACS Nano, 2014, 8(4): 3876-3883.

[74] HE Z, JIANG Y, MENG W, et al. Advanced LiTi ${ }_{2}\left(\mathrm{PO}_{4}\right)_{3} @ \mathrm{~N}$-doped carbon anode for aqueous lithium ion batteries. Electrochimica Acta, 2016, 222: 1491-1500.

[75] SUN D, TANG Y, HE K, et al. Long-lived aqueous rechargeable lithium batteries using mesoporous $\mathrm{LiTi}_{2}\left(\mathrm{PO}_{4}\right)_{3} @ \mathrm{C}$ anode. Scientific Reports, 2015, 5: 17452.

[76] XU T, ZHAO M, SU Z, et al. Nanostructured $\mathrm{LiTi}_{2}\left(\mathrm{PO}_{4}\right)_{3}$ anode with superior lithium and sodium storage capability aqueous electrolytes. Journal of Power Sources, 2021, 481: 229110.

[77] ROH H K, KIM H K, ROH K C, et al. $\mathrm{LiTi}_{2}\left(\mathrm{PO}_{4}\right)_{3} /$ reduced graphene oxide nanocomposite with enhanced electrochemical performance for lithium-ion batteries. RSC Advances, 2014, 4(60): 31672-31677.
[78] LIM C H, KANNAN A G, LEE H W, et al. A high power density electrode with ultralow carbon via direct growth of particles on graphene sheets. Journal of Materials Chemistry A, 2013, 1(20): 6183-6190.

[79] WANG H, YANG Y, LIANG Y, et al. $\mathrm{LiMn}_{1-x} \mathrm{Fe}_{x} \mathrm{PO}_{4}$ nanorods grown on graphene sheets for ultrahigh-rate-performance lithium ion batteries. Angewandte Chemie International Edition, 2011, 50(32): 7364-7368.

[80] ZHOU Z, LUO W, HUANG H, et al. LiTi $2_{2}\left(\mathrm{PO}_{4}\right)_{3} @$ carbon/ graphene hybrid as superior anode materials for aqueous lithium ion batteries. Ceramics International, 2017, 43(1): 99-105.

[81] ZHOU M, LIU L, YI L, et al. Synthesis of $\mathrm{LiTi}_{2}\left(\mathrm{PO}_{4}\right)_{3}$-acetylene black nanocomposites for lithium ion batteries by the polyvinyl alcohol assisted Sol-Gel method and ball-milling. Journal of Power Sources, 2013, 234: 292-301.

[82] LIU L, ZHOU M, WANG G, et al. Synthesis and characterization of $\mathrm{LiTi}_{2}\left(\mathrm{PO}_{4}\right)_{3} / \mathrm{C}$ nanocomposite as lithium intercalation electrode materials. Electrochimica Acta, 2012, 70: 136-141.

[83] WENG G M, TAM L Y S, LU Y C. High-performance $\mathrm{LiTi}_{2}\left(\mathrm{PO}_{4}\right)_{3}$ anodes for high-areal-capacity flexible aqueous lithium-ion batteries. Journal of Materials Chemistry A, 2017, 5(23): 11764-11771.

[84] HE Z, JIANG Y, ZHU J, et al. N-doped carbon coated $\operatorname{LiTi}_{2}\left(\mathrm{PO}_{4}\right)_{3}$ as superior anode using PANi as carbon and nitrogen bi-sources for aqueous lithium ion battery. Electrochimica Acta, 2018, 279: 279-288.

[85] ZHOU Z, XIANG A, XIA M, et al. Advanced $\operatorname{LiTi}_{2}\left(\mathrm{PO}_{4}\right)_{3}$ anode with high performance for aqueous rechargeable lithium battery. Ceramics International, 2018, 44(17): 21599-21606.

[86] YE J M, LI C M. Synthesis of $\mathrm{LiTi}_{2}\left(\mathrm{PO}_{4}\right)_{3} @$ carbon anode material with superior performance using beta-cyclodextrin as carbon sources. Ionics, 2020, 26(6): 2845-2853.

[87] BOUNAR N, BENABBAS A, ROPA P, et al. Synthesis and ionic conductivity of nasicon-structured $\mathrm{LiTi}_{2 x} \mathrm{Sn}_{x}\left(\mathrm{PO}_{4}\right)_{3}$ anode material for lithium-ion batteries. Advances in Materials and Processing Technologies, 2017, 3(3): 241-249.

[88] HE Z, JIANG Y, ZHU J, et al. Boosting the performance of $\mathrm{LiTi}_{2}\left(\mathrm{PO}_{4}\right)_{3} / \mathrm{C}$ anode for aqueous lithium ion battery by Sn doping on Ti sites. Journal of Alloys and Compounds, 2018, 731: 32-38.

[89] LIU N, HE Z, ZHANG X, et al. Synthesis and electrochemical properties of Na-doped $\mathrm{LiTi}_{2}\left(\mathrm{PO}_{4}\right)_{3} @$ carbon composite as anode for aqueous lithium ion batteries. Ceramics International, 2017, 43(14): 11481-11487.

[90] WANG H, ZHANG H, CHENG Y, et al. Rational design and synthesis of $\mathrm{LiTi}_{2}\left(\mathrm{PO}_{4}\right)_{3-x} \mathrm{~F}_{x}$ anode materials for high-performance aqueous lithium ion batteries. Journal of Materials Chemistry A, 2017, 5(2): 593-599.

[91] 张华民, 王怀清, 冯凯, 等. 一种阴离子掺杂的磷酸钛锂负极材 料及其制备和应用. ZL201610490240.3. 2016.6.29.

[92] LUO H, TANG Y, XIANG Z, et al. $\mathrm{Cl}^{-}$doping strategy to boost the lithium storage performance of lithium titanium phosphate. Frontiers in Chemistry, 2020, 8: 349.

[93] JIANG Z, LI Y H, HAN C, et al. Endowing $\mathrm{LiTi}_{2}\left(\mathrm{PO}_{4}\right)_{3} / \mathrm{C}$ with excellent electrochemical performances through rational crystal doping. Ceramics International, 2019, 45(17): 23406-23410. 